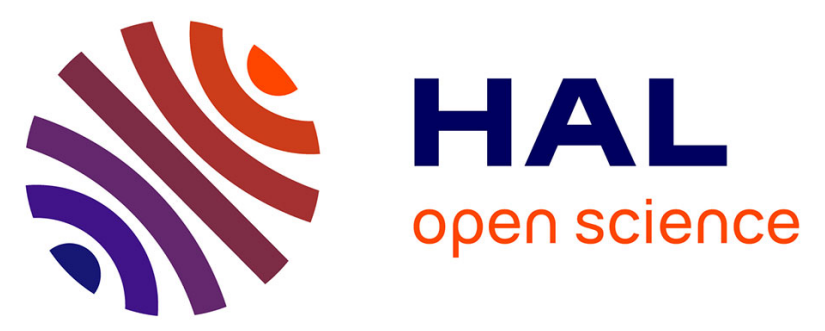

\title{
Ab Initio Study of Circular Dichroism and Circularly Polarized Luminescence of Spin-Allowed and Spin-Forbidden Transitions: From Organic Ketones to Lanthanide Complexes
}

Frédéric Gendron, Barry Moore Ii, Olivier Cador, Fabrice Pointillart, Jochen Autschbach, Boris Le Guennic

\section{To cite this version:}

Frédéric Gendron, Barry Moore Ii, Olivier Cador, Fabrice Pointillart, Jochen Autschbach, et al.. Ab Initio Study of Circular Dichroism and Circularly Polarized Luminescence of Spin-Allowed and SpinForbidden Transitions: From Organic Ketones to Lanthanide Complexes. Journal of Chemical Theory and Computation, 2019, 15 (7), pp.4140-4155. 10.1021/acs.jctc.9b00286 . hal-02179645

\section{HAL Id: hal-02179645}

\section{https://hal-univ-rennes1.archives-ouvertes.fr/hal-02179645}

Submitted on 19 Sep 2019

HAL is a multi-disciplinary open access archive for the deposit and dissemination of scientific research documents, whether they are published or not. The documents may come from teaching and research institutions in France or abroad, or from public or private research centers.
L'archive ouverte pluridisciplinaire HAL, est destinée au dépôt et à la diffusion de documents scientifiques de niveau recherche, publiés ou non, émanant des établissements d'enseignement et de recherche français ou étrangers, des laboratoires publics ou privés. 


\title{
Ab-initio Study of Circular Dichroism and Circularly Polarized Luminescence of Spin-allowed and Spin-forbidden Transitions: From Organic Ketones to Lanthanide Complexes
}

\author{
Frédéric Gendron, ${ }^{* a}$ Barry Moore II,${ }^{b}$ Olivier Cador, ${ }^{a}$ \\ Fabrice Pointillart, ${ }^{a}$ Jochen Autschbach* ${ }^{*}$ and Boris Le Guennic* $a$ \\ ${ }^{a}$ Univ Rennes, CNRS, ISCR (Institut des Sciences Chimiques de Rennes) \\ UMR 6226, F-35000 Rennes, France \\ ${ }^{b}$ Department of Chemistry, University at Buffalo \\ State University of New York, Buffalo, New York 14260-3000, U.S.A. \\ email: frederic.gendron@univ-rennes1.fr, jochena@buffalo.edu, \\ boris.leguennic@univ-rennes1.fr
}

May 23, 2019

\begin{abstract}
Complete and restricted active space self-consistent field (CAS- / RAS-SCF) wavefunction methods are applied for the calculation of circular dichroism (CD) and circularly polarized luminescence (CPL) of a series of molecules comprising four organic ketones, the chiral Cobalt(III) complex $\Lambda-\left[\mathrm{Co}(\mathrm{en})_{3}\right]^{3+}$ and the Europium(III) complex $\left[\mathrm{Eu}(\mathrm{DPA})_{3}\right]^{3-}$. The abinitio results are in good agreement with the experimental data and previous results obtained with Kohn-Sham density functional theory in the case of the spin-allowed transitions. CD and CPL properties are calculated ab-initio for the first time for the spin-forbidden transitions of both a transition metal and a lanthanide complex.
\end{abstract}

\section{Introduction}

The interest in chiral systems is tremendous because of their potential applications in a wide range of domains such as biochemistry, ${ }^{1}$ catalysis, ${ }^{2}$ pharmacology, ${ }^{3}$ nanotechnology ${ }^{4,5}$ or in the development of new photonic materials. ${ }^{6,7}$ The two enantiomers of a chiral molecule have many identical properties, but they also differ crucially in some aspects. For instance, the enantiomers interact 
differently with linearly and circularly polarized light, and they may absorb or emit photons preferentially with one sense of circular polarization. These are manifestations of the natural optical activity, i.e. the chiroptical properties exhibited by chiral compounds.

The enantiomers of a chiral molecule also interact differently with other chiral molecules and environments. The latter aspect is extremely important for drug design. For example, one enantiomer may exhibit the desired therapeutic behavior, while the other one may be inactive or even toxic. $^{8,9}$ Therefore, it is important to have access to methods that are able to provide reliable structural information and to characterize the absolute configuration of enantiomers. Here, chiroptical spectroscopic methods are the prime tools. ${ }^{10-13}$ For instance, the circular dichroism (CD) and circularly polarized luminescence (CPL) of electronic transitions can be used to obtain stereochemical information. $\mathrm{CD}$ is the difference in absorption between left- and right-circularly polarized light. CPL is the analog of CD in emission. Therefore, CD spectroscopy is well designed to provide structural information on the stereochemistry of the ground state, whereas CPL is better suited for the characterization of the excited states.

CD and CPL properties are often characterized experimentally by the dimensionless absorption and luminescence dissymmetry factors $g_{\text {abs }}$ and $g_{\text {lum }}$, respectively, which are defined as follows:

$$
g_{\mathrm{abs}}=\frac{\Delta \epsilon}{\epsilon}=\frac{\epsilon_{L}-\epsilon_{R}}{\frac{1}{2}\left(\epsilon_{L}+\epsilon_{R}\right)} \quad \text { and } \quad g_{\mathrm{lum}}=\frac{\Delta I}{I}=\frac{I_{L}-I_{R}}{\frac{1}{2}\left(I_{L}+I_{R}\right)}
$$

Here, $\epsilon_{L}$ and $\epsilon_{R}$ are the molar absorption coefficients for left- and right-circularly polarized light, respectively, and $I_{L}$ and $I_{R}$ are the corresponding luminescence intensities. From these definitions, it follows that the dissymmetry factors are within the range +2 and -2 , with the limits corresponding to an absolute preference of one circular polarization over the other. Typically, however, the CD and CPL of a transition is only a small fraction of the total absorption and emission intensity, respectively. Accordingly, solutions of organic molecules usually exhibit $|g|$ values on the order of $10^{-2}-10^{-5} \cdot 14,15$ Values of one order of magnitude larger are typical for transition metal complexes. ${ }^{16-21}$ The largest dissymmetry factors, within the range $0.1-1$, have been observed for transitions within the $4 \mathrm{f}$ manifold of lanthanide ( $\mathrm{Ln}$ ) complexes. ${ }^{22-27}$ Due to the weak crystal-field $(\mathrm{CF})$, these transitions are nearly electric-dipole $(E D)$ forbidden, but comparatively strongly magnetic-dipole $(M D)$ allowed, reinforcing the magnitude of the $g$ values. For instance, a $g_{\text {lum }}$ value of +1.38 was reported for the ${ }^{7} F_{1} \leftarrow{ }^{5} D_{0}$ transition of the Europium(III) complex $\mathrm{CsEu}[(+)-\mathrm{hfbc}]_{4} \cdot{ }^{28,29}$ It corresponds to the largest $|g|$ value measured to date for non-interacting molecules. Despite the widespread experimental use of these chiroptical spectroscopic techniques, the assignment and the interpretation of CD and CPL spectra in terms of individual transitions is rarely easy. Moreover, the magnitude and the sign of the $g$ values can be sensitive to the presence of a solvent and to conformational changes. Theoretical support is therefore highly needed in order to ascertain the mechanisms responsible for the optical activity of the studied systems.

From an ab-initio point of view, the calculation of the chiroptical parameters $g_{\text {abs }}$ and $g_{\text {lum }}$ requires the determination of the electronic spectrum and the $E D$ and $M D$ transition moments in the ground and excited state geometries, respectively. Kohn-Sham time-dependent density functional theory (TDDFT, usually with hybrid functionals), coupled-cluster theory (CC) with singles and doubles (CCSD), and other coupled-cluster variants, have so far been the methods of choice for this type of calculations. ${ }^{30-35}$ These methods have been thoroughly tested over the last two decades 
on a large set of organic compounds and on the spin-allowed transitions of some transition metal complexes. For instance, the experimental CD and CPL spectra of $d$-camphorquinone and $(S, S)$ trans- $\beta$-hydrindanone were nicely reproduced when using TDDFT calculations with inclusion of the vibronic effects. ${ }^{36}$ The results revealed that in both cases, the chiroptical properties arise from transitions between the $n$ and $\pi^{*}$ orbitals of the carbonyl groups. Subsequently, Pecul and Ruud ${ }^{37}$ applied TDDFT to a set of bicyclic ketones ${ }^{38}$ and showed that the differences in sign and magnitude between $g_{\text {abs }}$ and $g_{\text {lum }}$ are essentially related to the structural differences between the ground and excited states. Based on a similar set of compounds, it was shown that equation-of-motion CCSD (EOM-CCSD) and TDDFT with the CAM-B3LYP functional provided similar accuracy for $g_{\text {abs }}$ and $g_{\text {lum }}{ }^{39}$ while the popular B3LYP functional led to incorrect results in some of the emission properties.

There is also a substantial body of research on the calculation of spin-allowed electronic CD spectra of transition metal complexes, where TDDFT remains the main computational tool. See, e.g., References 33, 40 and 41 for reviews and References 19,42-50 for selected original studies. CPL calculations for transition metal complexes are scarce in comparison. A first attempt was made in 2008 by Coughlin et al. ${ }^{21}$ with the use of TDDFT in combination with an approximate formulation of $g_{\text {lum }}$, in order to determine the chiroptical properties associated to the $S_{0} \leftarrow T_{1}$ emission in a series of Iridium(III) complexes. Another attempt was made in 2013 with the use of multi-reference perturbative methods (CASSCF and CASPT2) on a cycloplatinated-[6]helicene compound. ${ }^{19}$ Despite the ability of lanthanide complexes to exhibit the largest dissymmetry factors, no ab-initio calculations of CD or CPL properties have been performed yet. Theoretical work on lanthanide complexes has so far relied on CF models ${ }^{51,52}$ in combination with the semiempirical Judd-Ofelt theory. ${ }^{53,54}$ Recently, such CF based models were used to simulate the CD spectrum $^{55}$ and the Raman optical activity ${ }^{56}$ of Ln-based compounds. TDDFT has been also used to calculate the CD spectrum of some Eu(III) and $\mathrm{Yb}$ (III) complexes, ${ }^{57-59}$ but these studies focused on the UV-Vis part of the energy spectrum which corresponds mostly to intra-ligand excitations. The lack of $a b$-initio data for the optical activity of metal-centered transitions arises from the added difficulty to take into account the effects from the spin-orbit coupling (SOC). For spin-forbidden transitions, the SOC is essential in bringing about the intensities, and for lanthanides, the SOC in the $4 \mathrm{f}$ and $5 \mathrm{~d}$ shells is sufficiently strong to alter the character of the transitions qualitatively.

Additionally, transition metal complexes with high symmetry, as well as most lanthanide complexes, afford degenerate electronic states that pose significant challenges for the aforementioned single-reference based methods that are mainly used for theoretical chiroptical studies. For instance, it is well known that the partially filled $4 f$ shells in the $\mathrm{Ln}$ (III) complexes leads to large static and dynamic electronic correlation effects that only multi-configurational approaches can tackle. ${ }^{60}$ Therefore, as an alternative to TDDFT and CC methods, we decided to perform $\mathrm{CD}$ and CPL calculations within the complete and restricted active space (CAS / RAS) ${ }^{61}$ selfconsistent field (SCF) multi-reference wavefunction framework, with SOC treated via state interaction (RASSI). ${ }^{62}$ In order to demonstrate that the approach is able to treat closed-shell organic molecules, transition metal complexes, and open-shell Ln-based systems with comparable accuracy, we performed calculations for representatives from each group, namely, four bicyclic ketones, the Cobalt(III) complex $\Lambda$ - $\left[\mathrm{Co}(\mathrm{en})_{3}\right]^{3+}$ (en = ethylenediamine), and the $\mathrm{Eu}(\mathrm{III})$ compounds $\Delta-, \Lambda-\left[\mathrm{Eu}(\mathrm{DPA})_{3}\right]^{3-}(\mathrm{DPA}=(2,6)$-pyridine-dicarboxylate $)$. 


\section{Theoretical and Computational Details}

\subsection{Kohn-Sham Density Functional Theory Calculations}

The Gaussian $09^{63}$ software package was used to perform structural optimizations of the investigated complexes. The ground state (GS) geometries were obtained using Kohn-Sham Density Functional Theory (DFT), whereas the structures of the corresponding excited states (ES) were optimized using the time-dependent DFT (TDDFT) response calculations. The GEDIIS/GDIIS algorithms were used for the geometry optimizations. ${ }^{64}$ The optimizations of the organic ketones employed either the Becke three-parameters exchange function in combination with the Lee-YangParr correlation functional (B3LYP ${ }^{65,66}$ ) with $20 \%$ of exact exchange or its Coulomb-attenuated version CAM-B3LYP. ${ }^{67}$ The basis set used for these optimizations was the correlation consistent basis set developed by Dunning and corresponded to a double- $\zeta$ contraction for the valence shell plus a polarization and diffuse functions (Aug-cc-pVDZ ${ }^{68}$ ). Starting from the X-ray diffraction structure, ${ }^{69}$ the GS structure of the Cobalt(III) tris-ethylenediamine complex $\Lambda$-[Co(en $\left.)_{3}\right]^{3+}$ was obtained from a DFT optimization using the B3LYP functional in combination with a triple- $\zeta$ plus polarization (TZVP) basis sets from the Ahlrichs group. ${ }^{70}$ In keeping with prior TDDFT studies of this complex ${ }^{42,43,45}$ the $(l e l)_{3}$ conformer of the complex was used for all calculations. In the case of the $\left[\mathrm{Eu}(\mathrm{DPA})_{3}\right]^{3-}$ complexes, the B3LYP functional was used for the optimization of the GS. In these calculations, a 28 -electron quasi-relativistic effective core potential $(\mathrm{ECP})^{71}$ along with a matching ECP28MWB-SEG valence basis set ${ }^{72}$ were used to replace the Eu core and mimic relativistic valence-shell effects. For the ligand atoms, the double- $\zeta$ basis sets with a polarization and diffuse functions from Dunning and Hay ${ }^{73}$ were used.

The CD and CPL dissymmetry factors were determined using TDDFT with the Amsterdam Density Functional software package (ADF2017) ${ }^{74-76}$ In these calculations, the influence of the functional was investigated by comparing the $\mathrm{PBE}^{77,78}$ (Perdew-Burke-Ernzrhof) generalized gradient approximation with its hybrid variant $\mathrm{PBE0} 0^{79,80}$ with $25 \%$ exact exchange $(\mathrm{eX})$ as well as with the functionals B3LYP ${ }^{65,66}$ and CAM-B3LYP ${ }^{67}$ (range-separated exchange with eX varying from 19 to $65 \%$ with increasing interelectronic distance). The basis set corresponded to the quadruple- $\zeta$ STO all-electron basis sets with four sets of polarization functions for all atoms (QZ4P). ${ }^{81}$ The rotatory strengths as well as the $E D$ moments were obtained within the Excitations module implemented in ADF. ${ }^{82,83}$ In the case of the organic compounds we used the nonrelativistic TDDFT module, ${ }^{84}$ while for the Cobalt complex we included scalar relativistic effects via the Zeroth-Order Relativistic Approximation (ZORA) ${ }^{85,86}$ Solvent effects were taken into account by using the Conductor-Like Screening Model (COSMO) with the dielectric constant of 78.4 to model water. ${ }^{87}$ The rotatory strengths were calculated using the dipole-length representation, which is known to be origin dependent. ${ }^{88}$ In order to evaluate the impact of the origin dependence with the chosen basis sets on the results, additional rotatory strength calculations were performed using the dipole-velocity representation. ${ }^{89}$ The comparison between the two formalisms (See Tables S2 and S3 of the SI) revealed very similar results between the two approaches, with calculated dissymmetry factors that only differ to the fourth digit in the case of the ketones. Therefore, only the results obtained with the dipole-length representation are provided in the article. The nature of the transitions involved in the CD and CPL spectra was analyzed with the help of the Natural Transition Orbitals (NTOs). ${ }^{90}$ The NTOs were then visualized with the graphical user interface 
of ADF.

\subsection{Wavefunction Calculations}

The multi-configurational and multi-reference calculations were carried out with the Molcas 8.0 software package. ${ }^{91}$ The calculations without spin-orbit coupling (denoted SR for 'scalar relativistic' in the following) employed the second-order Douglas-Kroll-Hess scalar relativistic Hamiltonian, ${ }^{92-95}$ in combination with the all-electron atomic natural orbital relativistically contracted (ANO-RCC) basis set from the Molcas library ${ }^{96-98}$ The basis sets were contracted to the triple- $\zeta$ plus polarization $(\mathrm{TZP})$ quality $(\mathrm{Eu}=25 \mathrm{~s} 22 \mathrm{p} 15 \mathrm{~d} 11 \mathrm{f} 4 \mathrm{~g} 2 \mathrm{~h} / 8 \mathrm{~s} 7 \mathrm{p} 4 \mathrm{~d} 3 \mathrm{f} 2 \mathrm{~g} 1 \mathrm{~h} ; \mathrm{Co}=21 \mathrm{~s} 15 \mathrm{p} 10 \mathrm{~d} 6 \mathrm{f} 4 \mathrm{~g} 2 \mathrm{~h} / 6 \mathrm{~s} 5 \mathrm{p} 3 \mathrm{~d} 2 \mathrm{f} 1 \mathrm{~g}$ ; C, N, O, = 14s9p5d3f2g/4s3p2d1f ; H = 8s4p3d1f/2s1p). State-averages calculations were performed either with the complete active space self-consistent field (CASSCF) approach or with the restricted active space self-consistent field (RASSCF) variant. ${ }^{61}$ Additionally, the dynamic correlation effects were treated using the complete active space perturbation theory at the second order (CASPT2) ${ }^{99}$ Solvent effects were included via the Conductor Polarizable Continuum Model (CPCM) as implemented in Molcas with the dielectric constant of water. ${ }^{100,101}$ The SOC was then introduced within a state interaction among the basis of calculated spin-orbit free states using the restricted active space state interaction (RASSI) approach. ${ }^{62}$ A local modification of the Molcas code was used to gain access to the different transition dipole moments calculated after treatment of the SOC interaction. ${ }^{102}$ The nature of the active spaces was confirmed by the visualization of the SR natural orbitals (NOs), using the graphical user interface of the ADF suite.

For the organic set of bicyclic ketones investigated here, the choice of the active space (AS) was driven by the NTO analysis performed at the TDDFT level on the same molecules. Depending on the nature of the ketones investigated, two different AS were used, namely CAS $(2,2)$ and $\mathrm{CAS}(4,3)$. The $\mathrm{CAS}(2,2)$ corresponds to two electrons in the oxygen lone pair $(n)$ and the $\mathrm{C}=\mathrm{O} \pi^{*}$ orbital, whereas the CAS $(4,3)$ corresponds to the $\operatorname{CAS}(2,2)$ space augmented by the doubly occupied $\pi$ orbital centered on the carbon double bond. The calculations employed the state-averaged formalism at the SR level by taking into account the three lowest singlet spin states in energy. For the CASPT2 calculations, an imaginary shift of 0.5 au in combination with the standard IPEA shift value of 0.25 au were used in order to avoid the presence of intruder states in the wavefunction. For the PT2-SO calculations, both the wave-functions and the energies from the SR CASPT2 calculations were employed.

For the $\Lambda$ - $\left[\mathrm{Co}(\mathrm{en})_{3}\right]^{3+}$ complex, the state-average calculations were performed by taking into account 7 singlet, 6 triplet and 5 quintet spin states. The AS used for the CASSCF and CASPT2 calculations corresponded to a $\operatorname{CAS}(10,12)$. It contains the five $3 d$ orbitals of the Co(III) center, plus the five pseudo- $4 d$ orbitals in order to take into account the double shell effect. ${ }^{103}$ Additionally, two doubly occupied ligand-based orbitals that can form a covalent $\sigma$-bond with the $3 d$ orbitals of formally $e_{g}$ symmetry in the $O_{h}$ parent symmetry were added in the AS. The actual symmetry of the complex is $D_{3}$, but it is often more convenient to refer to the parent symmetry instead. ${ }^{41}$ Additional ligand based orbitals were also included in the AS via RASCI calculations (i.e. RAS without orbital optimizations) on top of the CASSCF wavefunctions by allowing single and double excitations from an additional RAS1 space into the principal RAS2 space. This RAS1 space was formed by the $m$ highest-energy doubly occupied ligand-based orbitals, and the resulting AS are labelled as follows: $\mathrm{RAS}[n, 2,0, m, 12,0] \mathrm{CI}$, with $n$ corresponding to the number 
of active electrons and equal to $10+m \times 2$. The dynamic correlation effects were treated at the CASPT2 and RASPT2 level with an imaginary shift of 0.5 au. in combination with a modified IPEA shift of 0.5. The use of a larger value for the IPEA shift was found more appropriate to describe the relative energies of the different SR spin states in the case of transition metal complexes. ${ }^{104,105}$ For the PT2-SO calculations, the energies from the SR CASPT2 calculations and the wave-functions from the corresponding CASSCF / RASSCF calculations were employed in order to avoid symmetry breaking.

In the case of the $\Lambda$ - and $\Delta-\left[\operatorname{Eu}(\mathrm{DPA})_{3}\right]^{3-}$ complexes, several AS were investigated. Firstly, a CAS $(6,7)$ was used with the 6 electrons of the Eu(III) ion spanning the seven $4 f$ orbitals. Here, the state-average was performed on 7 septet, 5 quintet and 3 triplet spin states. The influence of the $5 d$ orbitals was investigated with a combination of RASSCF and RASCI calculations. A RAS[6,0,1,0,7,5]SCF calculation, including 112 septet spin states, was performed on top of the CAS(6,7)SCF wavefunction to optimize the $5 d$ orbitals. This RASSCF calculation allowed to create one particle in the RAS3 space containing the five $5 d$ orbitals of the Eu(III) ion. From these optimized orbitals, RAS[6,0,1,0,7,5]CI calculations were then performed for the 7 septet, 5 quintet and 3 triplet spin states. Using a similar strategy, we also investigated the influence of the $\pi-$ $\pi^{*}$ ligand-centered excitations. On top of the $\operatorname{CAS}(6,7)$ wave-function, a $\operatorname{RAS}[12,1,1,3,7,3] \mathrm{SCF}$ calculation was performed in which the RAS1 space was formed by three doubly occupied $\pi$ type orbitals centered on the ligands and the RAS3 space was formed with the corresponding three empty $\pi^{*}$ orbitals. From these optimized orbitals, RAS[12,1,1,3,7,3]CI calculations were then performed for 7 septet, 5 quintet and 3 triplet spin states. For the PT2-SO calculations, the energies from the SR CASPT2 calculations and the wave-functions from the corresponding CASSCF / RASSCF calculations were employed in order to avoid symmetry breaking.

\subsection{CD and CPL Theory}

The theory behind first-principles calculations of CD and CPL has been already extensively discussed, ${ }^{30,32,33}$ and therefore, only a few key points are discussed here. The probability of absorbing (or emitting) a photon with left vs. right circular polarization in a transition from an initial state $i$ to a final state $f$ is proportional to the rotatory strength $R_{i f}$. In the case of an isotropic sample, $R_{i f}$ corresponds to the imaginary part of the scalar product between the $E D$ transition moments $\boldsymbol{\mu}_{i f}$ and the $M D$ transition moments $\boldsymbol{m}_{f i}$, and is expressed in the length-dipole representation as follows:

$$
\begin{aligned}
\boldsymbol{R}_{i f} & =\operatorname{Im}\left[\boldsymbol{\mu}_{i f} \cdot \boldsymbol{m}_{f i}\right] \\
& =\operatorname{Im}\left[\left\langle i\left|e \sum_{n} \boldsymbol{r}_{n}\right| f\right\rangle \cdot\left\langle i\left|\frac{e}{2 m_{e} \cdot c} \sum_{n} \boldsymbol{L}_{n}+g_{e} \boldsymbol{S}_{n}\right| f\right\rangle\right]
\end{aligned}
$$

Here, the summations are over the Cartesian directions $n, g_{e} \simeq 2$ is the free electron $g$-factor, and $\boldsymbol{L}$ and $\boldsymbol{S}$ are the one-electron operators for the orbital and spin angular momentum, respectively. The spin contribution in Eq. 2 is zero if the states are spin-eigenfunctions. When the SOC becomes non negligible, the different states cannot anymore be described by pure spin-eigenfunctions and therefore, the transition matrix elements of the spin angular momentum operator may become non null. Moreover, the orbital angular momentum component in the magnetic transition dipole also generates intensity for a transition that is formally spin forbidden. The use of the dipole-length 
representation in Eq. 2 leads to origin-dependent results. However, our own test calculations (vide supra), as well as other works (e.g. recently in Refs. 106 and 107 in the context of X-ray absorption spectroscopy), have shown that the origin dependence is not pronounced with the use of a good quality basis sets and the centering of mass or centering of nuclear charge coordinates. For the metal-centered transitions in a metal complex, placing the (gauge) origin at the metal center is a straightforward choice. Additionally, we verified that a small displacement of the gauge origin does not drastically modify the results (see Table S8). The electric- and magnetic-transition dipole moments (TDMs) were obtained in atomic units and then converted to cgs units using a conversion factor $\alpha=471.44 \times 10^{40}$ as explained in Reference 108. The calculated $R_{i f}$ with Eq. 2 are therefore reported herein in cgs units of $10^{-40} \mathrm{esu}^{2} \mathrm{~cm}^{2}$. If the calculations of the TDMs are performed in the GS geometry, one obtains the CD of the absorption, whereas evaluation of the TDMs using the ES structures gives the CPL. It is worth reiterating that Eq. 2 assumes no contributions from the coupling between the $E D$ and the electric quadrupole $(E Q)$ because of an orientational average of this contribution. Such assumption is valid for an isotropic sample such as in solution, but does not hold in the case of a crystal. From there, the dissymmetry factor $g_{i f}$ for an isotropic sample is given by

$$
g_{i f}=\frac{4 \cdot R_{i f}}{D_{i f}}
$$

Here, $D_{i f}$ is a generalized 'dipole' strength and corresponds to the sum of the squared values of the $E D\left(\left|\boldsymbol{\mu}_{i f}\right|^{2}\right), M D\left(\left|\boldsymbol{m}_{i f}\right|^{2}\right)$ and $E Q\left(\left|\boldsymbol{Q}_{i f}\right|^{2}\right)$ transition moments as defined in Ref. 109. The reader is reminded that the magnitude of the $M D$ and $E Q$ moments are often found few order of magnitude smaller than the $E D$ moment in organic compounds. Therefore, Eq. 3 is often seen in the literature with only the $E D$ contribution in the denominator.

Using expressions from References 36 and 110, the absolute emission $(I)$ and CPL $(\Delta I)$ intensities are given by

$$
\begin{gathered}
I=\frac{4 \cdot E^{4}}{3 \cdot c^{3} \cdot \hbar^{4}}\left(\left|\boldsymbol{\mu}_{i f}\right|^{2}+\left|\boldsymbol{m}_{i f}\right|^{2}+\left|\boldsymbol{Q}_{i f}\right|^{2}\right) \\
\Delta I=\frac{16 \cdot E^{4}}{3 \cdot c^{3} \cdot \hbar^{4}}\left(R_{i f}\right)
\end{gathered}
$$

where $\hbar$ is the reduced Planck's constant, $c$ the speed of light and $E$ the energy of the given transition expressed in cgs units. In that case, the emission and CPL intensities are expressed in energy unit per time unit per molecule. Experimentally, it is difficult to know how many molecules emit. Therefore, the calculated emission and CPL intensities were normalized to arbitrary relative units after Gaussian-broadening with a $\sigma$ of $0.02 \mathrm{eV}$ (ca. $160 \mathrm{~cm}^{-1}$ ) in order to produce spectra for the $\left[\mathrm{Eu}(\mathrm{DPA})_{3}\right]^{3-}$ complex. 


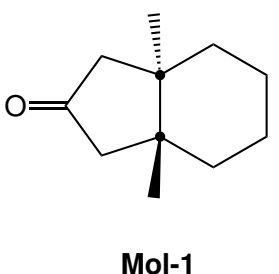

Mol-1

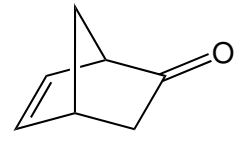

Mol-2

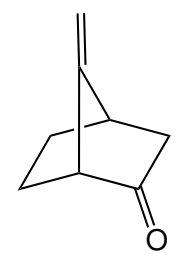

Mol-3

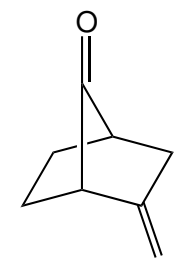

Mol-4

Figure 1: The set of organic molecules investigated here: Mol-1 $(S, S)$-trans- $\beta$-hydrindanone, Mol-2 $(1 R, 4 R)$-norbornenone, Mol-3 $(1 R)$-7-methylenebicyclo[2.2.1]heptan-2-one and Mol-4 (1S)-2-methylenebicyclo[2.2.1]heptan-7-one.

\section{Results and Discussion}

\subsection{Chiroptical Properties of Organic Systems}

\subsubsection{Circular Dichroism of bicyclic Ketones}

The calculations of chiroptical properties were first performed on a series of four bicyclic chiral ketones (Mol-1 - Mol-4) depicted in Figure 1. The calculated CD for these systems are given in Table 1 and are compared to the experimental values available and previous theoretical values. Overall, the rotatory strengths $(R)$ and the $E D$ moments obtained at the CAS level were found in reasonable agreement with the data obtained using other methods. In both compounds, the CAS calculations were able to reproduce the sign and the correct order of magnitude of the chiroptical properties. The largest discrepancies between the different methods are observed in the case of Mol-1, where the $g_{\text {abs }}$ calculated at the CASSCF and CASPT2 level are 2 to 3 times larger than those obtained at the TDDFT level (Table 1). Such differences can be explained by the degree of delocalization error present in the transition orbitals. ${ }^{111}$ Indeed, the CAS calculations tend to produce too localized $n$ and $\pi^{*}$ orbitals (see Figure S2), which leads to a decrease of the $E D$ moment and hence, an overestimation of $g_{\text {abs }}$. By comparison, the occupied NTOs from TDDFT calculations are less localized on the carbonyl bond, but the degree of delocalization error strongly depends on the amount of exact exchange (Table S1), and more generally on the functional used (Tables 1 and S2).

The amount of delocalization was also found crucial to properly reproduce the dissymmetry factors in Mol-2, Mol-3 and Mol-4. As previously noted successively by Autschbach and by Caricato with calculations of the optical activity in Mol-2, ${ }^{33,115,116}$ the magnitude of the CD parameters is strongly connected to the partial charge transfer that occurs in the GS between the carbonyl group and the $(\mathrm{C}=\mathrm{C})$ bond and vice versa. This charge transfer is characterized by the NTO analyses which revealed that the transitions corresponded to an admixture of $n \rightarrow \pi^{*}$ and $\pi(\mathrm{C}=\mathrm{C}) \rightarrow \pi^{*}$ excitations (Table S6). The $\pi(\mathrm{C}=\mathrm{C}) \rightarrow \pi^{*}$ contribution is also visible in Figure 2 and in Figures S3 - S5, where both the occupied and vacant NTOs exhibit lobes on the $(C=C)$ bond. The reader is reminded that using the same iso-surface value, the carbonyl transition of Mol-1 appeared perfectly localized. In agreement with Pecul et al. ${ }^{37}$ the dissymmetry factors obtained with the B3LYP functional were found closer to experiment than those obtained with CAM-B3LYP. This agreement is however only due to an overestimation of the $E D$ moments with 
the former functional because of a too small delocalization. Interestingly, the CAS calculations lied in between the TDDFT results. Indeed, the CAS ED moments were found in better agreement with the experimental values than the TDDFT ones. This is particularly true when using the PT2 corrected wave-funtions and energies, which increase the $\pi(\mathrm{C}=\mathrm{C})$ character of the wavefunction. Indeed, if the transition is mainly of $n \rightarrow \pi^{*}$ character $(\approx 85 \%)$ at the TDDFT level, the

Table 1: Principal calculated CD parameters for the lowest $n \rightarrow \pi^{*}$ transition in Mol-1, Mol-2, Mol-3 and Mol-4. The calculated vertical transition energies $(\Delta E$ in $\mathrm{eV})$, rotatory strengths $(R$ in $\left.\mathrm{esu}^{2} \mathrm{~cm}^{2}\right)$, electric dipole moments $\left(E D\right.$ in $\left.\mathrm{esu}^{2} \mathrm{~cm}^{2}\right)$ and absorption dissymmetry factors $\left(g_{\text {abs }}\right)$ are compared to previous calculated values and experimental data when available. ${ }^{a}$

\begin{tabular}{|c|c|c|c|c|c|c|}
\hline Compd & Level of Theory & $\overline{\Delta E}$ & $R\left(\times 10^{-40}\right)$ & $E D\left(\times 10^{-40}\right)$ & $g_{\mathrm{abs}}\left(\times 10^{-3}\right)$ & Ref. $^{b}$ \\
\hline \multirow[t]{7}{*}{ Mol-1 } & B3LYP & 4.21 & 15.89 & 217 & 292.8 & \\
\hline & CAM-B3LYP & 4.29 & 12.27 & 123 & 397.7 & \\
\hline & $\mathrm{CAS}(2,2) \mathrm{SCF}$ & 4.39 & 7.86 & 44 & 708.8 & \\
\hline & CAS(2,2)PT2 & 4.16 & 7.84 & 45 & 698.2 & \\
\hline & CAM-B3LYP & 4.23 & 12.10 & 123 & 394.2 & [39] \\
\hline & CCSD & 4.27 & 11.32 & 116 & 390.7 & [39] \\
\hline & Expt. & - & - & - & $\approx 200$ & [112], [113] \\
\hline \multirow[t]{8}{*}{ Mol-2 } & B3LYP & 4.09 & 57.68 & 4481 & 51.5 & \\
\hline & CAM-B3LYP & 4.24 & 45.26 & 2690 & 67.3 & \\
\hline & $\mathrm{CAS}(4,3) \mathrm{SCF}$ & 4.64 & 59.13 & 4842 & 48.8 & \\
\hline & CAS(4,3)PT2 & 3.91 & 49.07 & 3423 & 57.3 & \\
\hline & CAM-B3LYP & 4.18 & 45.57 & 2612 & 69.8 & [37] \\
\hline & CCSD & 4.26 & 34.06 & 1716 & 79.4 & [39] \\
\hline & $\mathrm{CC} 2$ & 4.30 & 42.50 & - & - & [114] \\
\hline & Expt. & $\approx 4.1$ & 51.10 & 3600 & 56.8 & [38] \\
\hline \multirow[t]{8}{*}{ Mol-3 } & B3LYP & 4.19 & 30.91 & 3003 & 41.2 & \\
\hline & CAM-B3LYP & 4.30 & 22.28 & 1551 & 57.4 & \\
\hline & CAS $(4,3) \mathrm{SCF}$ & 4.69 & 36.28 & 2259 & 64.2 & \\
\hline & $\mathrm{CAS}(4,3) \mathrm{PT} 2$ & 3.93 & 33.42 & 1814 & 73.7 & \\
\hline & CAM-B3LYP & 4.24 & 21.92 & 1527 & 57.4 & [37], [39] \\
\hline & CCSD & 4.31 & 18.74 & 1149 & 65.2 & [39] \\
\hline & $\mathrm{CC} 2$ & 4.17 & 23.40 & - & - & [114] \\
\hline & Expt. & $\approx 4.1$ & 20.50 & 1790 & 45.8 & [38] \\
\hline \multirow[t]{8}{*}{ Mol-4 } & B3LYP & 4.16 & -26.91 & 3207 & -33.5 & \\
\hline & CAM-B3LYP & 4.30 & -19.11 & 1480 & -51.6 & \\
\hline & CAS $(4,3) \mathrm{SCF}$ & 4.73 & -26.90 & 1620 & -66.4 & \\
\hline & CAS(4,3)PT2 & 3.93 & -24.51 & 1256 & -78.0 & \\
\hline & CAM-B3LYP & 4.23 & -18.61 & 1415 & -52.6 & [37], [39] \\
\hline & CCSD & 4.34 & -15.26 & 974 & -64.4 & [39] \\
\hline & $\mathrm{CC} 2$ & 4.37 & -20.30 & - & - & [114] \\
\hline & Expt. & $\approx 4.1$ & -15.20 & 1870 & -32.5 & {$[38]$} \\
\hline
\end{tabular}

$\bar{a}$ additional data with other functionals and using the dipole-velocity representation for the calculation of $R$ are available in Supporting Information. ${ }^{b}$ present calculations where no reference is given. 


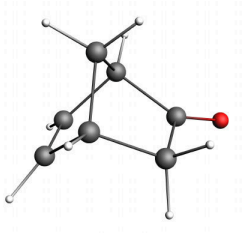

Mol-2

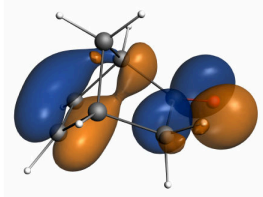

$\mathrm{NO}(\mathrm{C}=\mathrm{C})$

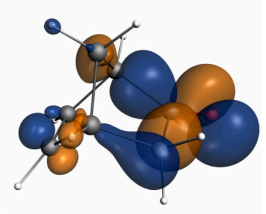

NTO Occ.

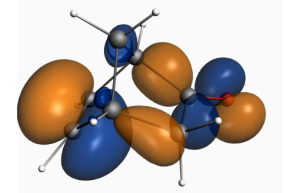

NO $(n)$

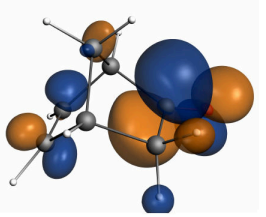

NTO Virt.

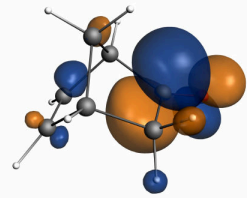

$\mathrm{NO}\left(\pi^{*}\right)$

Figure 2: (Top) Iso-surface ( \pm 0.03 a.u.) of the principal TDDFT (CAM-B3LYP) occupied (Occ.) and virtual (Virt.) natural transition orbitals (NTOs) for the GS $\rightarrow$ ES1 transition in Mol-2. (Bottom) Iso-surface ( \pm 0.03 a.u.) of the state-averaged natural orbitals (NOs) of Mol-2 from the CAS(4,3)SCF calculations.

$\pi(\mathrm{C}=\mathrm{C}) \rightarrow \pi^{*}$ character increases up to ca. $40 \%$ for the CASPT2 results. This strong admixture of configurations leads to an increase of the charge transfer toward the $\mathrm{C}=\mathrm{C}$ bond, and hence a decrease of the calculated $E D$ moments. On the other hand, larger transition $M D$ moments are calculated at the CAS level than at the DFT level, counterbalancing the reduction of the calculated ED moments.

\subsubsection{Circularly Polarized Luminescence of bicyclic Ketones}

The calculated CPL dissymmetry factors of the four ketones are given in Table 2. A relatively good agreement is obtained between the different levels of theory and with the experimental value in the case of Mol-1. A $g_{\text {lum }}$ of $47 \times 10^{-3}$ was calculated when using the CAM-B3LYP functional, while CCSD calculations gave a calculated dissymmetry factor of $35 \times 10^{-3} \mathrm{cgs}^{39}$ The difference between the two approaches results principally from an increase of the calculated $E D$ moment at the CCSD level. In comparison, the CAS calculations gave the largest $E D$ moment for this transition, leading to a theoretical $g_{\text {lum }}$ of $29 \times 10^{-3}$ in perfect agreement with the experiment. More interestingly, the CAS calculations were also able to reproduce the large decrease in magnitude between the CD and CPL dissymmetry factors. This behavior is explained principally by the large increase in magnitude of the $E D$ moments associated to the $n \leftarrow \pi^{*}$ transitions. Indeed, in the ES the $\mathrm{C}=\mathrm{O}$ bond is lengthened due to the population of an antibonding $\pi^{*}$ orbital, and the carbonyl group looses its planar configuration in favor of a pyramidal one. This lowering of symmetry in the ES leads to a stronger localization of the electron density on the carbonyl bond that reinforces the $E D$ moment.

A fairly good agreement was found between the different calculated $g_{\text {lum }}$ of Mol-2 and the experimental value. The Kohn-Sham results slightly overestimated the experimental value of 29 $\times 10^{-3}$ with calculated $g_{\text {lum }}$ of 32 and $40 \times 10^{-3}$ using the B3LYP and CAM-B3LYP functionals, respectively. On the other hand, the CAS calculations slightly underestimated the experimental dissymmetry factor with calculated values of 16 and $22 \times 10^{-3}$ at the CASSCF and CASPT2 
Table 2: Principal calculated CPL parameters for the lowest $n \leftarrow \pi^{*}$ transition in Mol-1, Mol-2, Mol-3 and Mol-4. The calculated vertical transition energies $(\Delta E$ in $\mathrm{eV})$, rotatory strengths $(R$ in $\left.\mathrm{esu}^{2} \mathrm{~cm}^{2}\right)$, electric dipole moments $\left(E D\right.$ in $\left.\mathrm{esu}^{2} \mathrm{~cm}^{2}\right)$ and absorption dissymmetry factors $\left(g_{\text {lum }}\right)$ are compared to previous calculated values and experimental data when available. ${ }^{a}$

\begin{tabular}{|c|c|c|c|c|c|c|c|}
\hline Compd & Level of Theory & Transition & $\Delta E$ & $R\left(\times 10^{-40}\right)$ & $E D\left(\times 10^{-40}\right)$ & $g_{\text {lum }}\left(\times 10^{-3}\right)$ & $\operatorname{Ref}^{b}$ \\
\hline \multirow[t]{7}{*}{ Mol-1 } & B3LYP & $\mathrm{GS} \leftarrow \mathrm{ES} 1$ & 3.19 & 11.40 & 826 & 55.2 & \\
\hline & CAM-B3LYP & $\mathrm{GS} \leftarrow \mathrm{ES} 1$ & 3.27 & 8.95 & 755 & 47.4 & \\
\hline & $\mathrm{CAS}(2,2) \mathrm{SCF}$ & $\mathrm{GS} \leftarrow \mathrm{ES} 1$ & 3.10 & 7.22 & 968 & 29.8 & \\
\hline & CAS(2,2)PT2 & $\mathrm{GS} \leftarrow \mathrm{ES} 1$ & 3.11 & 7.09 & 989 & 28.7 & \\
\hline & CAM-B3LYP & $\mathrm{GS} \leftarrow \mathrm{ES} 1$ & 3.21 & 8.85 & 749 & 47.2 & [39] \\
\hline & CCSD & $\mathrm{GS} \leftarrow \mathrm{ES} 1$ & 3.20 & 7.74 & 887 & 34.8 & [39] \\
\hline & Expt. & $\mathrm{GS} \leftarrow \mathrm{ES} 1$ & - & - & - & $\approx 27$ & [112], [113] \\
\hline \multirow[t]{8}{*}{ Mol-2 } & B3LYP & $\mathrm{GS} \leftarrow \mathrm{ES} 1$ & 2.80 & 32.02 & 4009 & 31.9 & \\
\hline & CAM-B3LYP & $\mathrm{GS} \leftarrow \mathrm{ES} 1$ & 2.92 & 27.59 & 2746 & 40.2 & \\
\hline & CAS $(4,3) \mathrm{SCF}$ & $\mathrm{GS} \leftarrow \mathrm{ES} 1$ & 2.79 & 28.58 & 7239 & 15.8 & \\
\hline & CAS $(4,3) \mathrm{PT} 2$ & $\mathrm{GS} \leftarrow \mathrm{ES} 1$ & 2.42 & 30.26 & 5474 & 22.1 & \\
\hline & CAM-B3LYP & $\mathrm{GS} \leftarrow \mathrm{ES} 1$ & 2.86 & 30.96 & 2699 & 45.9 & [37] \\
\hline & CCSD & $\mathrm{GS} \leftarrow \mathrm{ES} 1$ & 2.85 & 12.52 & 1338 & 37.4 & [39] \\
\hline & $\mathrm{CC} 2$ & $\mathrm{GS} \leftarrow \mathrm{ES} 1$ & 2.54 & 12.6 & & - & [114] \\
\hline & Expt. & $\mathrm{GS} \leftarrow \mathrm{ES} 1$ & - & - & - & 29.4 & {$[38]$} \\
\hline \multirow[t]{11}{*}{ Mol-3 } & B3LYP & $\mathrm{GS} \leftarrow \mathrm{ES} 1$ & 2.93 & 18.59 & 1210 & 61.4 & \\
\hline & & $\mathrm{GS} \leftarrow \mathrm{ES} 2$ & 2.94 & 0.04 & 4203 & 0.4 & \\
\hline & CAM-B3LYP & $\mathrm{GS} \leftarrow \mathrm{ES} 1$ & 3.01 & 12.91 & 1116 & 46.3 & \\
\hline & & $\mathrm{GS} \leftarrow \mathrm{ES} 2$ & 3.02 & -1.32 & 3068 & -1.7 & \\
\hline & CAS(2,2)SCF & $\mathrm{GS} \leftarrow \mathrm{ES} 1$ & 2.96 & 17.75 & 1914 & 37.1 & \\
\hline & & $\mathrm{GS} \leftarrow \mathrm{ES} 2$ & 2.99 & -1.66 & 4377 & -1.5 & \\
\hline & CAS(2,2)PT2 & $\mathrm{GS} \leftarrow \mathrm{ES} 1$ & 2.77 & 16.86 & 1899 & 35.5 & \\
\hline & & $\mathrm{GS} \leftarrow \mathrm{ES} 2$ & 2.77 & 0.75 & 4166 & 0.7 & \\
\hline & CCSD & $\mathrm{GS} \leftarrow \mathrm{ES} 1$ & 2.99 & 9.36 & 1311 & 28.5 & [39] \\
\hline & & $\mathrm{GS} \leftarrow \mathrm{ES} 2$ & 3.01 & -0.87 & 2884 & -1.2 & [39] \\
\hline & Expt. & & - & - & - & 15.7 & {$[38]$} \\
\hline \multirow[t]{11}{*}{ Mol-4 } & B3LYP & $\mathrm{GS} \leftarrow \mathrm{ES} 1$ & 2.20 & -13.49 & 1163 & -46.4 & \\
\hline & & $\mathrm{GS} \leftarrow \mathrm{ES} 2$ & 2.49 & 2.42 & 3992 & 2.4 & \\
\hline & CAM-B3LYP & $\mathrm{GS} \leftarrow \mathrm{ES} 1$ & 2.35 & -9.48 & 1372 & -27.6 & \\
\hline & $\rightarrow>$ & $\mathrm{GS} \leftarrow \mathrm{ES} 2$ & 2.57 & 3.20 & 3047 & 4.2 & \\
\hline & $\mathrm{CAS}(2,2) \mathrm{SCF}$ & $\mathrm{GS} \leftarrow \mathrm{ES} 1$ & 2.36 & -14.12 & 4249 & -13.3 & \\
\hline & $\sqrt{3}$ & $\mathrm{GS} \leftarrow \mathrm{ES} 2$ & 2.56 & 2.12 & 4069 & 2.1 & \\
\hline & $\mathrm{CAS}(2,2) \mathrm{PT} 2$ & $\mathrm{GS} \leftarrow \mathrm{ES} 1$ & 1.96 & -14.93 & 3392 & -17.6 & \\
\hline & & $\mathrm{GS} \leftarrow \mathrm{ES} 2$ & 2.10 & 1.27 & 4107 & 1.2 & \\
\hline & CCSD & $\mathrm{GS} \leftarrow \mathrm{ES} 1$ & 2.42 & -6.82 & 1788 & -15.2 & [39] \\
\hline & & $\mathrm{GS} \leftarrow \mathrm{ES} 2$ & 2.60 & 3.41 & 2995 & 4.5 & [39] \\
\hline & Expt. & & - & - & - & $<3$ & {$[38]$} \\
\hline
\end{tabular}

$\bar{a}$ additional data with other functionals and using the dipole-velocity representation for the calculation of $R$ are available in Supporting Information. ${ }^{b}$ present calculations where no reference is given. 
level, respectively. Interestingly, CCSD produced a $g_{\text {lum }}$ of similar magnitude, ${ }^{39}$ but with calculated $R$ and $E D$ moment much smaller than those obtained with the TDDFT or CAS calculations. Similarly to Mol-1, the dissymmetry factor of Mol-2 decreases when going from absorption to emission. In that case, the reduction is less pronounced in magnitude and follows the reduction of $R$. As explained by Caricato, ${ }^{117}$ the magnitude of $R$ depends on the angle formed by the $E D$ and $M D$ moments, which is related to the dihedral angle formed by the $\mathrm{C}=\mathrm{O}$ and $\mathrm{C}=\mathrm{C}$ bonds. The rotatory strength is maximized when the two groups are coplanar, whereas $R$ becomes null when they are out-of-plane. In Mol-2, the dihedral angle between the two fragments decreases from 142 to $120^{\circ}$ when going from the GS to the ES. This decrease of co-planarity goes along with a decrease of charge transfer in the ES. Indeed, the $\pi(\mathrm{C}=\mathrm{C}) \rightarrow \pi^{*}$ character decreases from 40 to $28 \%$ when going from the GS to the ES at the CASPT2 level.

Previous TDDFT and CCSD calculations performed on Mol-3 and Mol-4 have shown that for these two compounds the potential energy surface of the ES has two minima that exhibit very different chiroptical properties. ${ }^{37,39}$ These two minima are labelled ES1 and ES2 in the following discussion and correspond respectively to structures in which the carbonyl bond has bent away and toward the $\mathrm{C}=\mathrm{C}$ bond upon the $n \rightarrow \pi^{*}$ excitation (see Figures S4 and S5). In Mol-3, ES1 exhibits a relatively large and positive calculated $g_{\text {lum }}$, whereas the dissymmetry factor associated to ES2 is small and even negative. The best agreement with the experimental value was obtained at the CCSD level with a calculated $g_{\text {lum }}$ of $28 \times 10^{-3}$ with the ES 1 structure. ${ }^{39}$ On the other hand, the TDDFT method gave much larger values with $g_{\text {lum }}=46$ and $61 \times 10^{-3}$ when using the CAMB3LYP and B3LYP functionals, respectively. The CAS calculations were found lying in between these two methods with calculated dissymmetry factors of 37 and $35 \times 10^{-3}$ at the CASSCF and CASPT2 level, respectively. In Mol-4, ES1 exhibits a sizable negative dissymmetry factor that ranges from -13 to $-46 \times 10^{-3}$ when going from CASSCF to B3LYP results, respectively, strongly overestimating the experimental value. Despite much larger vertical transition energies, the calculated $g_{\text {lum }}$ associated to the ES2 structure were found closer to the experiment with $g_{\text {lum }}=$ 2 and $1 \times 10^{-3}$ at the CASSCF and CASPT2 level, respectively. As explained originally by Pecul et al. ${ }^{37}$ the CPL properties in Mol-3 and Mol-4 are related to the excited state ordering and to this purpose, the adiabatic transition energies must be taken into account. The ES1 structure is favored with the B3LYP functional for both Mol-3 and Mol-4 (see Table S5), however, the ES2 structure in Mol-4 becomes almost iso-energetic to ES1 using the CAM-B3LYP functional, and hence, increasing the population of the ES2 structure. It is worth mentioning that previous adiabatic CAM-B3LYP and CC calculations have found the ES2 structure more stable than ES1. ${ }^{37,39}$

\subsection{CD parameters in a Cobalt(III) Complex}

The CD spectrum of the Cobalt(III) complex $\Lambda$ - $\left[\mathrm{Co}(\mathrm{en})_{3}\right]^{3+}$ has been extensively studied both experimentally and theoretically since the early work of Kobayashi and Mathieu. ${ }^{118,119}$ The rotatory strengths for the low-energetic part of the spectrum were characterized with measurements performed on the crystal structure at $80 \mathrm{~K}$ and in aqueous solution at room temperature. ${ }^{16,120}$ A first characteristic of the spectrum is the two CD bands around $21000 \mathrm{~cm}^{-1}$ that exhibit surprisingly large $R$ with opposite sign ( $R \approx \pm 55 \times 10^{-40} \mathrm{cgs}$ ). These bands were assigned to transitions into the excited singlet states spanning the $E$ and $A_{2}$ irreducible representations ("irreps") of the $D_{3}$ point group. ${ }^{121-124}$ In order to rationalize the magnitude and the sign of these $R$, several mod- 

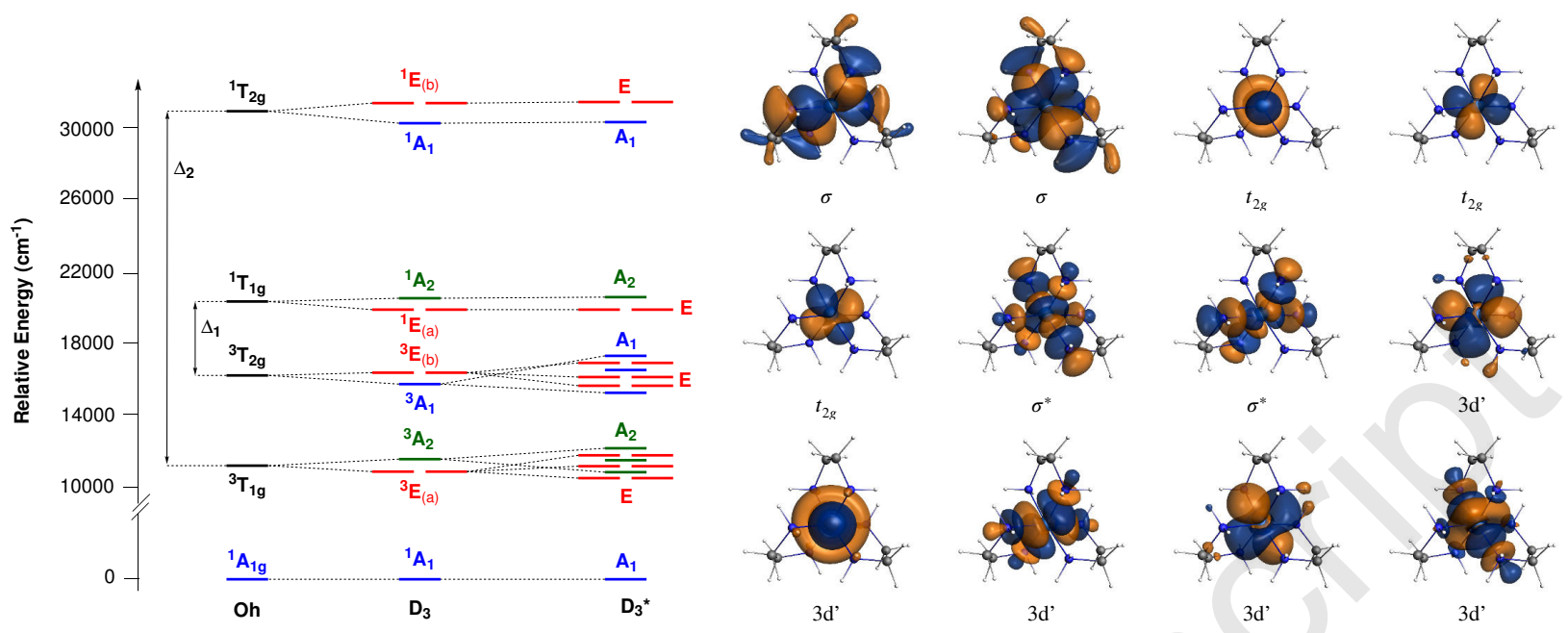

Figure 3: (left) Energy state diagram for the lowest states of $\Lambda$ - $\left[\mathrm{Co}(\mathrm{en})_{3}\right]^{3+}$ extracted from CAS(10,12)SCF-SR/SO calculations performed on the experimental structure taken from Ref 69. The scalar (SR) and spin-orbit (SO) states of $\Lambda$ - $\left[\mathrm{Co}(\mathrm{en})_{3}\right]^{3+}$ are labelled in the $D_{3}$ and $D_{3}^{*}$ symmetry point groups, respectively. The parent SR states of $O_{h}$ symmetry are given for comparison. (right) Plot of the iso-surfaces $( \pm 0.03 \mathrm{au}$.) of the state-averaged natural orbitals.

els based on CF or ligand-field (LF) theory were developed during the 1960s and 1970s. ${ }^{125-129}$ Despite the large amount of analysis, none of these models were able to fully reproduce the experimental data as they were designed to describe only metal-centered $3 d$ transitions. The importance of the metal-ligand interactions on the CD spectrum were then demonstrated by using restricted Hartree-Fock theory in the early 1990s, ${ }^{130}$ and fully rationalized by Ziegler and coworkers with the use of TDDFT calculations. ${ }^{42-45,131}$ Another important feature in the CD spectrum is the presence of weakly intense CD bands corresponding to formally spin-forbidden transitions into the lowest excited triplet states. ${ }^{120}$ To the best of our knowledge, the intensities of these transitions have not been studied yet with the help of ab-initio calculations, principally because of the necessity to include the SOC effects. This work represents therefore a first attempt to calculate the optical activity of these spin-forbidden $3 d$ transitions.

\subsubsection{Electronic Structure of $\Lambda-\left[\mathrm{Co}(\mathrm{en})_{3}\right]^{3+}$}

The electronic structure of $\Lambda$ - $\left[\mathrm{Co}(\mathrm{en})_{3}\right]^{3+}$ has been already described using CF theory, ${ }^{121-124}$ and different flavors of DFT and TDDFT calculations. ${ }^{42-45,131}$ Its principal features are summarized in Figure 3 with a state energy diagram obtained from the CAS $(10,12)$ SCF-SR/SO calculations and plots of the iso-surfaces of the natural orbitals generated in this active space. At the SR level, the GS corresponds to the non-degenerate singlet ${ }^{1} A_{1}$ in the $D_{3}$ symmetry point group. This state derives from an ${ }^{1} A_{1 g}$ singlet in the parent $O_{h}$ symmetry point group and corresponds formally to a $t_{2 g}^{6} e_{g}^{0}$ electronic configuration. As shown in Figure 3, a strong covalent interaction of $\sigma$ character takes place between the metal-centered orbitals spanning the $e_{g}$ irreps and two ligandcentered orbitals of same symmetry. The bonding combinations of this interaction are doubly occupied and are labelled $\sigma$, whereas the antibonding combinations (labelled $\sigma^{*}$ ) are vacant and 
destabilized in energy. On the other hand, the $3 d$ orbitals of formally $t_{2 g}$ symmetry remain nonbonding in character, and are therefore doubly occupied in the GS. The first set of ESs is found lying around $11500 \mathrm{~cm}^{-1}$ above the GS and corresponds to a doubly degenerate triplet state ${ }^{3} E$ and a non-degenerate triplet ${ }^{3} A_{2}$. These SR triplet states derive from the splitting by the trigonal field of the parent ${ }^{3} T_{1 g}$ state and correspond to a $t_{2 g}^{5} e_{g}^{1}$ electronic configuration. The next triplet states are calculated at $16000 \mathrm{~cm}^{-1}$ above the GS and derive from the parent ${ }^{3} T_{2 g}$ state. Here, the triplet states span the ${ }^{3} E$ and ${ }^{3} A_{1}$ irreps of the $D_{3}$ point group. Finally, the lowest singlet states are localized around 20000 and $31000 \mathrm{~cm}^{1}$ above the GS and derive from the parent triply degenerate singlet states ${ }^{1} T_{1 g}$ and ${ }^{1} T_{2 g}$, respectively. Introduction of the SOC does not drastically change the electronic structure. The energy of the SR singlet states is barely affected by the SOC, whereas the components of the SR triplets ${ }^{3} E$ and ${ }^{3} A_{1}$ on one side, and the components of ${ }^{3} E$ and ${ }^{3} A_{2}$ on the other side, are allowed to mix with each others, leading to a small energetic splitting of the triplet states.

\subsubsection{CD parameters of $\Lambda-\left[\operatorname{Co}(e n)_{3}\right]^{3+}$}

The calculated energies, $E D$ moments and $R$ associated to the lowest spin-allowed transitions of $\Lambda$ $\left[\mathrm{Co}(\mathrm{en})_{3}\right]^{3+}$ are given in Table 3. Experimentally, the lowest spin-allowed transitions, measured at ca. $21000 \mathrm{~cm}^{-1}$, correspond to excitations into the excited singlet states $E$ and $A_{2}$, with $R$ values for the crystal structure of +59.9 and $-55.7 \times 10^{-40} \mathrm{cgs},{ }^{132}$ respectively. A relatively good agreement with the experimental data was obtained at the TDDFT level. For instance, the use of the CAM-B3LYP functional leads to calculated $R$ of +42.79 and $-33.17 \times 10^{-40}$ cgs for the $E$ and $A_{2}$ transitions, respectively. As shown in Tables S11 and S12, the magnitude of the calculated $R$ is strongly functional and solvent dependent, while the energy of the transitions is always slightly overestimated. Nevertheless, our calculated values are of the same order of magnitude with those previously calculated with a similar approach. ${ }^{42,45}$ At the CASPT2 level, the $E$ and $A_{2}$ bands are calculated at 19950 and $20867 \mathrm{~cm}^{-1}$, respectively, in good agreement with the crystal structure data. However, the $R$ associated to these bands are relatively small with calculated values of 8.50

Table 3: Calculated energies $\left(\Delta E, \mathrm{~cm}^{-1}\right)$, electric dipole moment $\left(E D, \times 10^{-40} \mathrm{esu}^{2} \mathrm{~cm}^{2}\right)$ and rotatory strengths $\left(R, \times 10^{-40} \mathrm{esu}^{2} \mathrm{~cm}^{2}\right)$ for the formally lowest spin-allowed transitions in $\Lambda$ $\left[\mathrm{Co}(\mathrm{en})_{3}\right]^{3+}$. The CAS and RAS results are compared to the TDDFT and experimental data when available. ${ }^{a}$

\begin{tabular}{|c|c|c|c|c|c|c|c|c|c|c|c|}
\hline & CAS & 10,12 & PT2 & RAS[1 & 2,0 & ,0]CI-PT2 & & $\overline{\text { M-B3 }}$ & & & \\
\hline & $\Delta E$ & $E D$ & $R$ & $\Delta E$ & $E D$ & $R$ & $\Delta E$ & $E D$ & $R$ & $\Delta E$ & $R$ \\
\hline$E$ & 19950 & 15 & 8.50 & 19549 & 111 & 28.46 & 22643 & 323 & 42.79 & $20760^{b}$ & $+59.9^{b}$ \\
\hline & & & & & & & & & & $21300^{c}$ & $+4.80^{c}$ \\
\hline$A_{2}$ & 20867 & 22 & -9.04 & 20546 & 176 & -25.13 & 22598 & 382 & -33.17 & $20990^{b}$ & $-55.7^{b}$ \\
\hline & & & & & & & & & & $23700^{c}$ & $-0.4^{c}$ \\
\hline$A_{1}$ & 29007 & 0 & 0.00 & 28653 & 0 & 0.00 & 29117 & 0 & 0 & & \\
\hline$E$ & 29862 & 0 & -0.01 & 29441 & 91 & -0.08 & 29526 & 295 & -0.24 & $29400^{c}$ & $+0.70^{c}$ \\
\hline
\end{tabular}


and $-9.01 \times 10^{-40} \mathrm{cgs}$ for $E$ and $A_{2}$, respectively. Such small calculated values can be explained by the too localized character of the orbitals included in the active space. Indeed, as originally described by Mason and Richardson, ${ }^{123,133}$ the rotatory strengths of the $d$ - $d$ transitions may gain in magnitude via a dynamic coupling that involves a metal-ligand polarization mechanism. Similarly, Fan and Ziegler have shown more recently that the intensity of the calculated $R$ at TDDFT level is related to the metal-ligand mixing in the valence orbitals. ${ }^{45}$ To this purpose, we increased the size of our active space by including $m$ doubly occupied orbitals that are principally localized on the diamine ligands (see Section 2 for more details). The results are presented in Table 3 for the RAS[18,2,0,4,12,0] active space, while additional results can be found in Tables S7 - S10 of the SI. It is clearly visible in Table 3 that the introduction of the ligand based orbitals in the active space leads to a large increase of the calculated $R$ associated to the $A_{1} \rightarrow E$ and $A_{1} \rightarrow A_{2}$ transitions, with calculated values of +28.46 and $-25.13 \times 10^{-40} \mathrm{cgs}$. The ligand-based orbitals responsible for this increase in magnitude of $R$ are shown in Figure S6 and correspond to $\sigma$-type bonding metal-ligand orbitals with some $4 p$ Cobalt character. The excitations from these orbitals into the valence metal-centered orbitals leads to a mixing of gerade and ungerade configurations in the wavefunction that strongly increases the magnitude of the $E D$ transition moments, and hence the rotatory strengths (see Tables S7 and S10).

The second set of excited singlet states is calculated around ca. $29000 \mathrm{~cm}^{-1}$ above the GS from the CASPT2 calculations. As expected from the selection rules of group theory, the $A_{1} \rightarrow A_{1}$ does not exhibit any optical activity as it is both formally $E D$ and $M D$ forbidden. On the other hand, the $A_{1} \rightarrow E$ transition is permitted and an extremely small and negative $R$ is obtained at the CAS level. The increase of the active space leads again to a sizable increase in magnitude of the $E D$ moment and for the largest RAS space investigated here, a $R$ of $-0.08 \times 10^{-40} \mathrm{cgs}$ is calculated. For comparison, a better agreement is obtained at the TDDFT level for the excitation energies, while a slightly more negative $R$ is calculated (see Tables S11 and S12). The difference of sign with the experimental data is attributed to structural changes between the fully characterized crystal structure used for the calculations and the admixture of structures present in solution. For instance, the RAS calculations performed on the optimized structure of $\Lambda$ - $\left[\mathrm{Co}(\mathrm{en})_{3}\right]^{3+}$ gave a small and positive $R$ of $0.33 \times 10^{-40} \mathrm{cgs}$ (see Table S10), in good agreement with the $0.70 \times 10^{-40} \mathrm{cgs}$ measured in solutions.

The calculated CD parameters for the spin-forbidden transitions are given in Table 4 and are compared to the experimental data. The magnitude of the calculated $R$ is a direct consequence of the SOC and is proportional to the triplet-singlet energy gaps $\Delta_{1}$ and $\Delta_{2}$ shown on Figure 3 . Indeed, in the $O_{h}$ symmetry point group the ${ }^{3} T_{1 g}$ triplets are allowed to mix with the ${ }^{1} T_{2 g}$ states, whereas the ${ }^{3} T_{2 g}$ states couple with the ${ }^{1} T_{1 g}$ states. At the CAS(10,12)PT2-SO level, the calculated $R$ for the states with ${ }^{3} T_{1 g}$ parentage are almost null, while very small $R$ are calculated for some of the states with ${ }^{3} T_{2 g}$ parentage. Introduction of ligand-to-metal excited configurations in the wavefunction via the RASCI calculations leads to a slight improvement in the magnitude of the calculated transition moments. This is particularly true for the excited states of ${ }^{3} \boldsymbol{T}_{2 g}$ parentage, where a sizable increase of the $E D$ moment is obtained at the RASCI level. Numerical agreement with the experimental data is not achieved, but the calculations reproduce the difference in magnitude of $R$ between the two sets of transitions.

The origin of the small calculated $R$ for these transitions are threefold. Firstly, the CAS and RAS calculations overestimate the SR $\Delta_{1}$ and $\Delta_{2}$ energy gaps, which reduce the admixture of 
singlet states in the triplet wavefunctions via the SOC, and hence reduce the magnitude of the transition dipole moments. This argument is particularly true for the transitions involving the state of ${ }^{3} T_{2 g}$ parentage, as the ${ }^{1} T_{1 g}$ states are closely lying above (see Figure 3 ). Indeed, if one recalculates the CD parameters of $\Lambda$ - $\left[\mathrm{Co}(\mathrm{en})_{3}\right]^{3+}$ by using the RAS[18,2,0,4,12,0]CI wavefunctions in combination with the experimental excitation energies, the calculated $R$ for the first two transition in ${ }^{3} T_{2 g}$ increases from ca. $|0.4|$ to $|2.1| \times 10^{-40} \mathrm{cgs}$, respectively (see Table S9). Secondly, the use of the effective one-electron Atomic Mean Field Spin-Orbit (AMFI) operator for the treatment of the SOC can lead to a lost of accuracy in the calculations of the transition dipole moments for spin-forbidden transitions. For instance, the calculated $E D$ moments and $R$ of singlet-triplet transitions in a set of ketones were found almost twice smaller when using the AMFI operator compared to a full two-electron spin-orbit operator. ${ }^{134}$ Finally, it is worth mentioning that a more accurate calculation of the $R$ should require to take into account the influence of the vibronic and Jahn-Teller effects which in both cases, by lowering the symmetry, would bring additional intensity in the $E D$ transition moments.

\subsection{CPL parameters of an Europium(III) Complex}

The $\left[\mathrm{Eu}(\mathrm{DPA})_{3}\right]^{3-}$ complex represents a good model compound for the calculation of CPL parameters in Ln-based systems. Chemically, the main interest of this system resides in the fact that the DPA ligands, which generate the $\Lambda$ or $\Delta$ helical arrangement, are easily substituted in order to take advantage of the antenna effect, and hence, tune the chiroptical properties. ${ }^{135}$ Therefore, the $\left[\mathrm{Eu}(\mathrm{DPA})_{3}\right]^{3-}$ complex is often seen as the molecular backbone for the design of chiral biological

Table 4: Calculated energies $\left(\Delta E, \mathrm{~cm}^{-1}\right)$, electric dipole moment $\left(E D, \times 10^{-40} \mathrm{esu}^{2} \mathrm{~cm}^{2}\right)$, rotatory strengths $\left(R, \times 10^{-40} \mathrm{esu}^{2} \mathrm{~cm}^{2}\right)$ for the formally lowest spin-forbidden transitions in $\Lambda$ $\left[\mathrm{Co}(\mathrm{en})_{3}\right]^{3+}$. The CAS and RAS results are compared to the TDDFT and experimental data when available. ${ }^{a}$

\begin{tabular}{cccccccccc}
\hline & \multicolumn{6}{c}{ CAS $(10,12) \mathrm{PT} 2$} & \multicolumn{2}{c}{$\mathrm{RAS}[18,2,0,4,12,0] \mathrm{CI}-\mathrm{PT} 2$} & \multicolumn{2}{c}{ Expt. } \\
& & $\Delta E$ & $E D$ & $R$ & $\Delta E$ & $E D$ & $R$ & $\Delta E$ & $R$ \\
\hline$\left({ }^{3} T_{1 g}\right)$ & $E$ & 12010 & 0.65 & 0.01 & 11610 & 0.48 & 0.01 & 13500 & $-0.005^{b}$ \\
& $A_{2}$ & 12119 & 0.24 & 0.04 & 11722 & 0.36 & 0.04 & & $+0.068^{c}$ \\
& $E$ & 12226 & 0.77 & 0.05 & 11825 & 0.98 & 0.06 & & \\
& $A_{2}$ & 12458 & 0.08 & -0.02 & 12057 & 0.08 & -0.04 & & \\
& $E$ & 12793 & 0.15 & -0.07 & 12396 & 0.069 & -0.05 & & \\
& $A_{2}$ & 13001 & 0.07 & 0.00 & 12605 & 0.06 & 0.00 & & \\
$\left({ }^{3} T_{2 g}\right)$ & $A_{1}$ & 16048 & 0.27 & -0.13 & 15649 & 2.00 & -0.35 & 17000 & $+0.07^{b}$ \\
& $E$ & 16122 & 1.29 & 0.23 & 15722 & 2.24 & 0.32 & & $+0.32^{c}$ \\
& $E$ & 16622 & 0.34 & -0.18 & 16166 & 0.20 & 0.13 & & \\
& $A_{1}$ & 16831 & 0.39 & 0.03 & 16363 & 0.30 & 0.02 & & \\
& $A_{1}$ & 17109 & 0.41 & 0.06 & 16827 & 0.28 & -0.04 & & \\
& $E$ & 17178 & 0.12 & -0.03 & 16765 & 0.69 & 0.09 & & \\
\hline
\end{tabular}

${ }^{a}$ The state labels correspond to those of the $D_{3}^{*}$ double point group as used in Figure 3 and derives from the scalar ${ }^{3} T_{1 g}$ and ${ }^{3} T_{2 g}$ state of $O_{h}$ parentage. ${ }^{b}$ Experimental data in solution at room temperature extracted from Ref 120. ${ }^{c}$ Experimental data on crystal structure at $80 \mathrm{~K}$ extracted from Ref 120. 
Figure 4: (left) Energy state diagram for the lowest states of $\Lambda$-[Eu(DPA $\left.)_{3}\right]^{3-}$ extract from CAS(6,7)PT2-SO calculations performed on the optimized structure. The SO states of $\Lambda$ $\left[\mathrm{Eu}(\mathrm{DPA})_{3}\right]^{3-}$ are labelled using the $D_{3}^{*}$ double point group of symmetry. (right) Plot of the isosurfaces $( \pm 0.03 \mathrm{au}$.) of the state-averaged natural orbitals included in the RAS $[6,0,2,0,7,5]$ space.

sensors. ${ }^{24,136}$ Theoretically, the principal difficulty in the CPL calculations of Ln complexes arises in the correct estimation of the ED TDMs associated to the $4 f$ transitions. It has been shown recently that the use of the CASSCF approach, with an AS containing the seven $4 f$ orbitals, is able to recover at least some parts of the $E D$ moments. ${ }^{137,138}$ To obtain qualitative agreement with the experiment, one must also take into account the different mechanisms that drive the intensity of the chiroptical activity, namely the static and dynamic coupling. ${ }^{27}$ The former corresponds to the mixing of the $4 f^{n}$ electronic configurations with the excited configurations $4 f^{(n-1)} 5 d^{1}$ in order to break the parity rule. This mechanism is strongly related to potential covalent effects between the Ln center and the coordinating ligands. On the other hand, the dynamic coupling mechanism corresponds to the coupling between the quadrupole moment of the Ln center with the induced dipole moment of the ligands generated by the oscillating light. ${ }^{139}$ This mechanism corresponds to the mixing of the $4 f^{n}$ wavefunction with additional configurations resulting from intra-ligand excitations. 


\subsubsection{Electronic Structure of $\left[\mathrm{Eu}(\mathrm{DPA})_{3}\right]^{3-}$}

The electronic structure of $\left[\mathrm{Eu}(\mathrm{DPA})_{3}\right]^{3-}$ has been already extensively studied both theoretically and experimentally, ${ }^{56,140-143}$ and therefore, is only briefly reminded. As described on the left hand side of Figure 4, the $4 f^{6}$ electronic configuration of the $\mathrm{Eu}(\mathrm{III})$ free ion gives rise via the Coulomb repulsion to a septet ground term ${ }^{7} F$ that is well separated from the lowest excited term ${ }^{5} D$. Introduction of the SOC leads to a splitting of these terms into the Russel-Saunders levels characterized by their total angular momentum $J$. For the free ion, the ground level corresponds to the non-degenerate ${ }^{7} F_{0}$, and the excited levels ordering goes with the increasing value of $J$. Similarly, the excited quintet term ${ }^{5} D$ is split into five ${ }^{5} D_{J}$ levels with $J=0$ being the lowest in energy. The trigonal CF generated by the DPA ligands then lifts the degeneracy of these levels and mixes the different $m_{J}$ states. In Figure 4, the states of $\left[\mathrm{Eu}(\mathrm{DPA})_{3}\right]^{3-}$ are labelled using the irreps of the $D_{3}^{*}$ symmetry double point group. The free ion ${ }^{7} F_{0}$ gives the non-degenerate $A_{1}$ state as the GS in the complex. The trigonal environment splits the ${ }^{7} F_{1}$ level into 3 states spanning the $E$ and $A_{2}$ irreps, whereas the degeneracy of the ${ }^{7} F_{2}$ level is lifted into $2 E \oplus A_{1}$ states.

The optical activity in $\mathrm{Eu}(\mathrm{III})$-based complexes is principally due to transitions from the excited ${ }^{5} D_{0}$ state into the different CF states of the ${ }^{7} F_{J}$ levels. ${ }^{135,143} \mathrm{In}\left[\mathrm{Eu}(\mathrm{DPA})_{3}\right]^{3-}$, the excited $A_{1}$ state of ${ }^{5} D_{0}$ parentage is calculated at 20690 and $17760 \mathrm{~cm}^{-1}$ above the GS at the SCF-SO and PT2-SO level, respectively. For comparison, this emitting state was characterized experimentally between 17180 and $17300 \mathrm{~cm}^{-1}$ above the GS, ${ }^{144-146}$ revealing the importance to take into account the dynamic correlation effects in the calculations to properly reproduce the electronic spectra. At the CASPT2-SO level, the calculated energies for the ${ }^{7} F_{1} \leftarrow{ }^{5} D_{0},{ }^{7} F_{2} \leftarrow{ }^{5} D_{0}$ and ${ }^{7} F_{3} \leftarrow{ }^{5} D_{0}$ emissions are of ca. 17375,16610 and $15597 \mathrm{~cm}^{-1}$, respectively. These calculated values slightly overestimate the experimental emission energies that were characterized with CPL measurements. ${ }^{147}$ Indeed, the experimental CPL spectrum of $\Lambda-\mathrm{Eu}(\mathrm{DPA})_{3}{ }^{3-}$ exhibits positive CPL bands at 16949 and $15455 \mathrm{~cm}^{-1}$, corresponding to the emissions into the ${ }^{7} F_{1}$ and ${ }^{7} F_{3}$ states, respectively, whereas a negative CPL band, assigned to the ${ }^{7} F_{2} \leftarrow{ }^{5} D_{0}$ transitions, was measured at $16260 \mathrm{~cm}^{-1}$. The calculated ${ }^{7} F_{4} \leftarrow{ }^{5} D_{0}$ transition energy is of ca. $14494 \mathrm{~cm}^{-1}$ in perfect agreement with experimental the positive and negative CPL bands measured at ca. $14400 \mathrm{~cm}^{-1}$.

\subsubsection{CPL parameters of $\Lambda$ - and $\Delta-\operatorname{Eu}(\mathrm{DPA})_{3}{ }^{3-}$}

The calculated emission and CPL spectra of $\Lambda$-[Eu(DPA $\left.)_{3}\right]^{3-}$ with the different active spaces investigated are shown in Figure 5, while the corresponding spectra for the $\Delta$ enantiomer are shown in Figure S8 of the SI. The reader is reminded that the emission $(I)$ and the CPL $(\Delta I)$ intensities are proportional to the calculated dipole moments and rotatory strengths, respectively, via the Equations 4 and 5 given in Section 2.3. At the CAS(6,7)PT2-SO level, only two bands are visible in the emission spectrum. The strongest band in emission is calculated around 17500 $\mathrm{cm}^{-1}$ and corresponds to the ${ }^{7} F_{1} \leftarrow{ }^{5} D_{0}$ transitions. These transitions are intense due to their predominant $M D$ character. A weaker band is calculated at ca. $14500 \mathrm{~cm}^{-1}$ and corresponds to the ${ }^{7} F_{4} \leftarrow{ }^{5} D_{0}$ transitions, which contain enough $E D$ character to be visible in the spectrum. As shown in Table S14, the ${ }^{7} F_{2,3} \leftarrow{ }^{5} D_{0}$ transitions have too small transition moments to be characterized in the calculated emission spectrum. The corresponding calculated CPL spectrum reveal a more complex structure with the presence of additional bands. The most intense band in emission $\left({ }^{7} F_{1} \leftarrow{ }^{5} D_{0}\right)$ is now split into two bands with a very intense and positive one, corresponding to 
CAS(6,7)PT2-SO
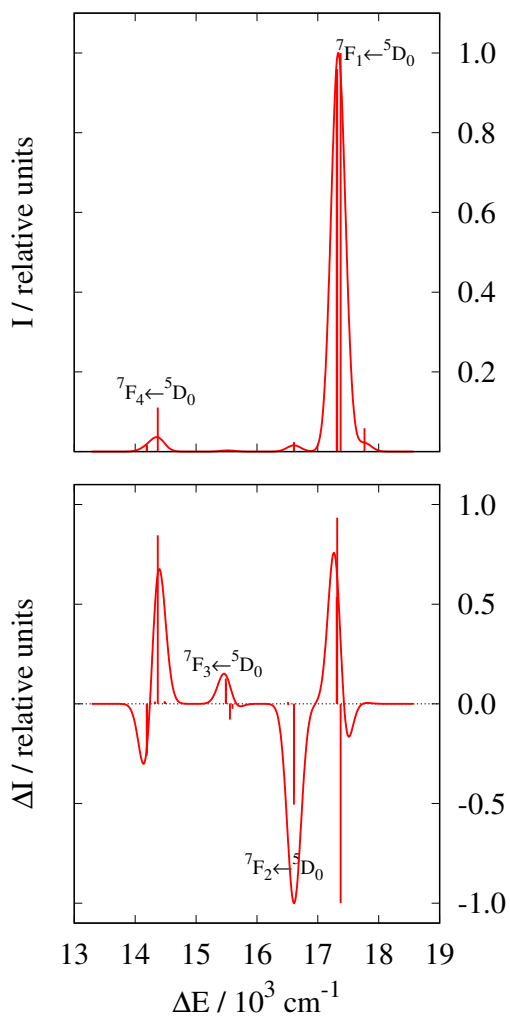
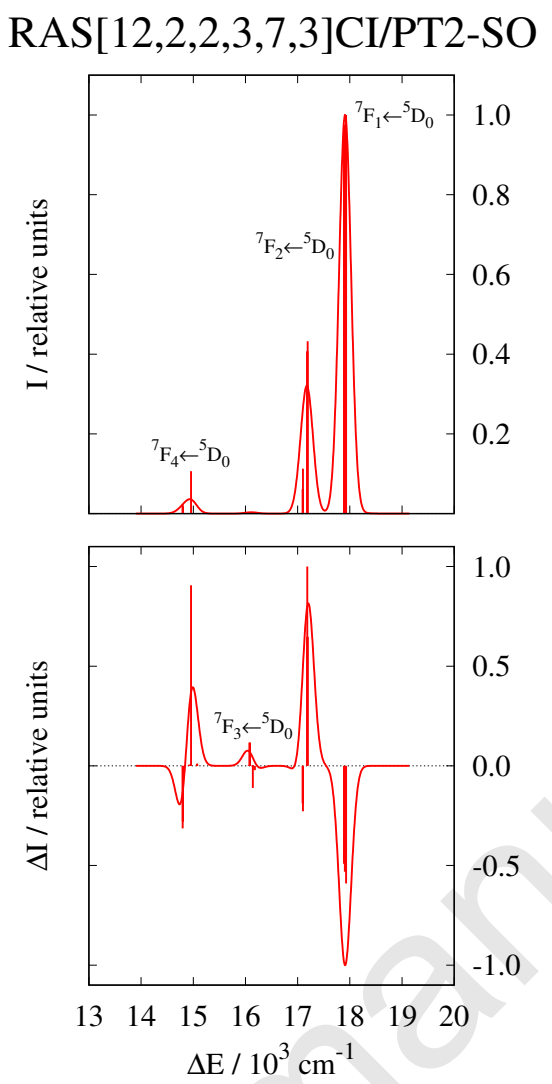

RAS $[6,0,2,0,7,5] \mathrm{CI} / \mathrm{PT} 2-\mathrm{SO}$
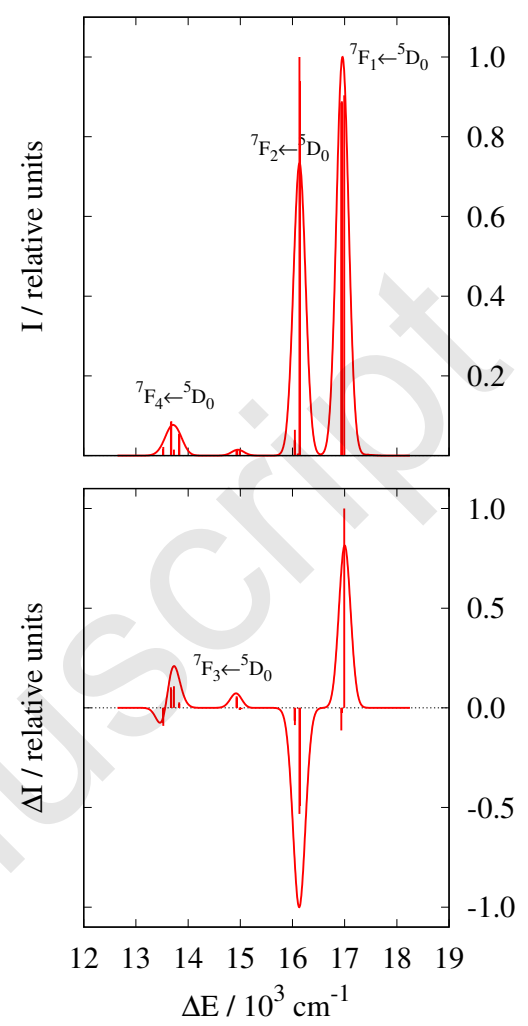

Figure 5: Normalized emission $(I)$ and CPL $(\Delta I)$ spectra in $\Lambda$-[Eu(DPA $\left.)_{3}\right]^{3-}$ for the ${ }^{7} F_{J} \leftarrow$ ${ }^{5} D_{0}(J=0-4)$ transitions obtained at the CAS(6,7)PT2-SO, RAS[12,2,2,3,7,3]CI/PT2-SO and RAS[6,0,2,0,7,5]CI/PT2-SO levels. The numerical values used for these spectra are given in Table S13. The dipole moments used for the emission plots take into account the $E D, M D$ and $E Q$ transition moments.

$E \leftarrow A_{1}$ transitions, and a negative band corresponding to the $A_{2} \leftarrow A_{1}$ transition. A second band in CPL appears at $16610 \mathrm{~cm}^{-1}$ and corresponds to an emission into one of the $E$ states of the ${ }^{7} F_{2}$ level. Similarly, emissions into the $E$ and $A_{2}$ states of the ${ }^{7} F_{4}$ level generate a negative and positive signal, respectively in the CPL spectrum. It is important to note that both the calculated dipole moments and rotatory strengths of the ${ }^{7} F_{3} \leftarrow{ }^{5} D_{0}$ transitions are extremely small (Table S14), but the associated dissymmetry factors are excessively large. This behavior only results from the definition of $g$ in Equation 3 and a division by almost zero.

Introduction of the ligand-based $\pi$ and $\pi^{*}$ orbitals at the RAS[12,2,2,3,7,3]CI/PT2 level strongly affects the shape of the calculated spectra. In the emission spectrum, an additional band appears at ca. $17100 \mathrm{~cm}^{-1}$ and corresponds to emissions into the $E$ states of the ${ }^{7} F_{2}$ level. The increase of the emission intensity is characterized in Table S14 by the large increase in magnitude of the calculated dipole moments. For instance, the calculated dipole moment of the first $E \leftarrow A_{1}$ emission increases from 18 to $31710^{-44} \mathrm{cgs}$ when going from CAS(6,7)PT2-SO to RAS[12,2,2,3,7,3]CI/PT2$\mathrm{SO}$, respectively. It is important to mention that this increase in magnitude results only from the $E D$ contributions and not from the $M D$ ones. This is in good agreement with the experimental observations and the hypersensitivity of the ${ }^{7} F_{2} \leftarrow{ }^{5} D_{0}$ emissions to the environment. Overall, the 
emission intensities of the other transitions are not affected by the presence of the ligand orbitals. The shape of the CPL spectrum is also strongly modified when the ligand $\pi$ orbitals are included in the active space with an inversion of sign of the two strongest CPL bands. Indeed, at the RAS level the ${ }^{7} F_{1} \leftarrow{ }^{5} D_{0}$ transitions afford a negative CPL band whereas the ${ }^{7} F_{2} \leftarrow{ }^{5} D_{0}$ transitions leads to a positive CPL band.

The presence of the Eu(III) $5 d$ orbitals in the RAS[6,0,2,0,7,5] space also modify the shape of the calculated spectra. Similarly to the introduction of the ligand-based $\pi$ orbitals, the mixing of the $4 f^{6} \mathrm{CFs}$ with the $4 f^{6} 5 d^{1} \mathrm{CFs}$ leads to a strong increase in magnitude of the ${ }^{7} F_{2} \leftarrow{ }^{5} D_{0}$ emission band, and in a lesser extent of the ${ }^{7} F_{4} \leftarrow{ }^{5} D_{0}$ one, when compared to the CAS spectrum. This increase in magnitude is found much more pronounced than the one calculated with the ligand $\pi$ orbitals. For instance, the calculated dipole moment of the excitation into the lowest E state of the ${ }^{7} F_{2}$ level increases from 317 to $107610^{-44}$ cgs when using the RAS[12,2,2,3,7,3] and RAS $[6,0,2,0,7,5]$ spaces, respectively (see Table S14). On the other hand, the magnitude of the calculated dipole moment for the emissions into the ${ }^{7} F_{1}$ and ${ }^{7} F_{3}$ levels is much less affected by the $5 d$ orbitals because of their $E D$ forbidden character. The CPL spectrum calculated at the RAS[6,0,2,0,7,5]CI/PT2-SO level is comparatively similar to the one obtained at the CAS(6,7)PT2-SO level. The ${ }^{7} F_{1} \leftarrow{ }^{5} D_{0}$ and ${ }^{7} F_{2} \leftarrow{ }^{5} D_{0}$ transitions afford positive and negative CPL bands, respectively. The large increase of the $E D$ moment for these transitions leads to a strong increase of the calculated $R$, and hence of the CPL intensities. The bands associated to emissions into the ${ }^{7} F_{3}$ and ${ }^{7} F_{4}$ levels afford in both cases positive CPL bands of similar intensities to the one calculated at the CAS level.

The comparison with the CPL measurements performed in solution by Riehl and coworker, ${ }^{147}$ reveals that the best agreement in term of energy and sign of the CPL bands is obtained when the $\mathrm{Eu}$ (III) $5 d$ orbitals are included in the active space. Indeed, CPL bands with positive, negative and positive sign were experimentally assigned to the ${ }^{7} F_{1} \leftarrow{ }^{5} D_{0},{ }^{7} F_{2} \leftarrow{ }^{5} D_{0}$ and ${ }^{7} F_{3} \leftarrow{ }^{5} D_{0}$ transitions, respectively, whereas the ${ }^{7} F_{4} \leftarrow{ }^{5} D_{0}$ transitions exhibited two CPL bands with positive and negative $\Delta I$. Introduction of the ligand $\pi$ orbitals in the active space does not allow to produce the correct signs of the CPL bands and has a much smaller influence on the magnitude of the calculated dipole moments. These results suggest, surprisingly, that the effects from the static coupling are more important in $\left[\mathrm{Eu}(\mathrm{DPA})_{3}\right]^{3-}$ than those from the dynamic coupling. However, in order to achieve quantitative numerical agreement with the experimental data, it is likely necessary to combine the two strategies and design much larger active spaces. Active spaces of the envisioned size are out of reach when using the RAS method used in this work, but will likely be feasible with spin-orbit matrix product state (MPS-SO) techniques. ${ }^{148}$

\section{Conclusions and Perspectives}

The CD and CPL properties of four organic ketones, the $\Lambda-\left[\mathrm{Co}(\mathrm{en})_{3}\right]^{3+}$ complex, and of the $\left[\mathrm{Eu}(\mathrm{DPA})_{3}\right]^{3-}$ enantiomers were investigated using CASSCF/PT2 and RASSCF/PT2 wavefunctions. The use of these multi-reference approaches, in combination with a treatment of the spinorbit coupling a posteriori via the RASSI method, allowed us to characterize the chiroptical properties for both the spin-allowed and spin-forbidden transitions of the systems investigated.

The study of the organic ketones have shown that our WFT approach performs in comparable 
agreement with the mono-determinental methods (TDDFT or CC) that are usually used in the case of spin-allowed transitions. In that case, the reduction of the dissymmetry factors associated to the $n \leftrightarrow \pi^{*}$ transitions between absorption and emission was properly reproduced by our calculations and explained by the large increase in magnitude of the ED moment in emission. Secondly, a satisfactory agreement was obtained between the TDDFT and WFT results for the calculated rotatory strengths of the spin-allowed $3 d-3 d$ transitions in $\Lambda$ - $\left[\operatorname{Co}(\mathrm{en})_{3}\right]^{3+}$. This agreement was achieved at the RAS level by including in the wavefunction additional configurations corresponding to excitations from the two highest occupied ligand-based orbitals into the Cobalt centered $3 d$ orbitals. This mixing of configurations led to a large increase of the calculated ED moments, and hence, of the rotatory strengths. At our best level of calculations, the calculated $R$ were found slightly underestimating the magnitude of the experimental values characterized for the crystal structure. Using the same approach, the CD properties of the spin-forbidden transitions were also investigated. At the RAS level, the numerical agreement was not achieved but the calculated $R$ were found in the right order of magnitude. Additionally, it was shown that the magnitude of the calculated $R$ is strongly dependent from the SOC between the SR excited triplet and singlet spin states and the energy gap between these states.

Finally, the emission and CPL properties associated to the $4 f-4 f$ excitations in the $\left[\mathrm{Eu}(\mathrm{DPA})_{3}\right]^{3-}$ complex were also calculated using WFT. The magnitude of the calculated emission and CPL spectra was found to be very sensitive to the nature of the chosen active space. The introduction of the ligand based $\pi-\pi^{*}$ excitations led to an expected increase of the emission intensities of the $E D$-allowed ${ }^{7} F_{2} \leftarrow{ }^{5} D_{0}$ transitions because of a large increase of the calculated $E D$ moments. However, introduction of these ligand configurations led to drastic changes in the CPL spectrum with an inversion of sign for the two largest bands in opposition with the experimental characterizations. The introduction of the $4 f^{5} 5 d^{1}$ configurations in the wavefunction also led to a very large increase in magnitude of the calculated ED moments, and hence of the emission intensities. Additionally, the signs and the energies of the calculated CPL bands were found in good agreement with the experimental data. These results indicate that the introduction of the $4 f^{5} 5 d^{1}$ configurations in the wavefunction are necessary in order to calculate CPL spectra that qualitatively reproduce the experimental ones. However, in order to reach quantitative agreement, introduction of both the $5 d$ and ligand $\pi$ orbitals in the active space would also be necessary. For this purpose, the density matrix renormalization group-based MPS-SO approach, ${ }^{148,149}$ is a method of choice and will be investigated in follow-up work.

\section{Acknowledgments}

The authors acknowledge support from the European Research Council under the European Union's Horizon 2020 research and innovation program (ERC-CoG MULTIPROSMM, Grant agreement $\mathrm{N}^{\circ}$ 725184). FG and BLG thank the French GENCI/IDRIS-CINES centers for high-performance computing resources. FG and BLG acknowledge the Stratégie d'Attractivité Durable (SAD18006 - LnCPLSMM) for financial support. BA thanks Rennes Métropole for a travel fellowship. JA acknowledges support from the National Science Foundation, Grant $N^{\circ}$ CHE-1560881. We thank the Center for Computational Research (CCR) at the University at Buffalo for providing computational resources. 


\section{Supporting Information}

Additional data regarding the KS-TDDFT and WFT CD and CPL parameters for Mol-1 - Mol4, natural transition orbitals, WFT and TDDFT CD parameters of $\Lambda$ - $\left[\mathrm{Co}(\mathrm{en})_{3}\right]^{3+}$ and emission and CPL spectra of $\Delta$-[Eu(DPA $\left.)_{3}\right]^{3-}$. This material is available free of charge via the Internet at http://pubs.acs.org.

\section{References}

[1] Heffern, M. C.; Matosziuk, L. M.; Meade, T. J. Lanthanide Probes for Bioresponsive Imaging Chem. Rev. 2014, 114, 4496-4539.

[2] Noyori, R. Asymmetric Catalysis: Science and Opportunites (Nobel Lecture) Angew. Chem. Int. Ed. 2002, 41, 2008-2022.

[3] McConnell, O.; Bach, A.; Balibar, C.; Byrne, N.; Cai, Y.; Carter, G.; Chlenov, M.; Di, L.; Fan, K.; Goljer, I. et al. Enantiomeric separation and determination of absolute stereochemistry of asymmetric molecules in drug discovery-Building chiral technology toolboxes Chirality 2007, 19, 658-682.

[4] Zhang, J.; Albelda, M. T.; Liu, Y.; Canary, J. W. Chiral nanotechnology Chirality 2005, $17,404-420$.

[5] Brandt, J. R.; Salerno, F.; Fuchter, M. J. The Added Value of Small-Molecule Chirality in Technological Applications Nature Reviews Chemistry 2017, 1, 0045.

[6] Huck, N. P. M.; Jgar, W. F.; de Lange, B.; Feringa, B. L. Dynamic Control and Amplification of Molecular Chirality by Circularly Polarized Light Science 1996, 273, 1696-1688.

[7] Haupert, L. M.; Simpson, G. J. Chirality in Nonlinear Optics Annu. Rev. Phys. Chem. 2009, 60, 345-365.

[8] Nguyen, L. A.; He, H.; Pham-Huy, C. Chiral Drugs: An Overview Int. J. Biomed. Sci. 2006, 2, 85-100.

[9] Chhabra, N.; Aseri, M. L.; Padmanabhan, D. A Review of Drug Isomerism and Its Signifiance Int. J. Appl. Basic Med. Res. 2013, 3, 16-18.

[10] Polavarapu, P. L. Renaissance in chiroptical spectroscopic methods for molecular structure determination Chem. Rec. 2007, 7, 125-136.

[11] Polavarapu, P. L. Molecular Structure Determination Using Chiroptical Spectroscopy: Where We May Go Wrong? Chirality 2012, 24, 909-920.

[12] Berova, N.; Polavarapu, P. L.; Nakanishi, K.; Woody, R. W., Eds.; Comprehensive Chiroptical Spectroscopy; volume 1: Instrumentation, Methodologies, and Theoretical Simulations John Wiley \& Sons: New York, 2012. 
[13] Berova, N.; Polavarapu, P. L.; Nakanishi, K.; Woody, R. W., Eds.; Comprehensive Chiroptical Spectroscopy; volume 2: Applications in Stereochemical Analysis of Synthetic Compounds, Natural Products, and Biomolecules John Wiley \& Sons: New York, 2012.

[14] Sánchez-Carnero, E.; Agarrabeita, A. R.; Moreno, F.; Maroto, B. L.; Muller, G.; Ortiz, M.; de la Moya, S. Curcularly Polarized Luminescence from Simple Organic Molecules Chem. Eur. J. 2015, 21, 13488-13500.

[15] Tanak, H.; Inoue, Y.; Mori, T. Circularly Polarized Luminescence and Circular Dichroisms in Small Organic Molecules: Correlation between Excitation and Emission Dissymmetry Factors Chem. Photo Chem. 2018, 2, 386-402.

[16] McCaffery, A. J.; Mason, S. F. The Electronic Spectra, Optical Rotatory Power and Absolute Configuration of Metal Complexes. The Dextro-tris (Ethylenediamine)Cobalt(III) ion Mol. Phys. 1963, 6, 359-371.

[17] Mason, S. F.; Norman, B. J. Outer-Sphere Co-ordination and Optical Activity in TransitionMetal Complexes Chem. Commun. 1965, 335-336.

[18] Richardson, F. S. Theory of Optical Activity in the Ligand-Field Transitions of Chiral Transition Metal Complexes Chem. Rev. 1979, 79, 17-36.

[19] Shen, C.; Anger, E.; Srebro, M.; Vanthuyne, N.; Deol, K. K.; Jefferson, T. D.; Muller, G.; Williams, J. A. G.; Toupet, L.; Roussel, C. et al. Straightforward access to mono- and bis-cycloplatinated helicenes that display circularly polarized phosphorescence using crystallization resolution methods Chem. Sci. 2014, 5, 1915-1927.

[20] Zhang, X.-P.; Chang, V. Y.; Liu, J.; Yang, X.-L.; Huang, W.; Li, Y.; Li, C.-H.; Muller, G.; You, X.-Z. Potential Switchable Circularly Polarized Luminescence from Chiral Cyclometalated Platinum(II) Complexes Inorg. Chem. 2015, 54, 143-152.

[21] Coughlin, F. J.; Westrol, M. S.; Oyler, K. D.; Byrne, N.; Kraml, C.; Zysman-Colman, E.; Lowry, M. S.; Bernhard, S. Synthesis, Separation, and Circularly Polarized Luminescence Studies of Enantiomers of Iridium(III) Luminophores Inorg. Chem. 2008, 47, 2039-2048.

[22] Riehl, J. P.; Richardson, F. S. Circularly Polarized Luminescence Spectrsocopy Chem. Rev. 1986, $86,1-16$.

[23] Bruce, J. I.; Parker, D.; Lopinski, S.; Peacock, R. Survey of Factors Determining the Circularly Polarised Luminescence of Macrocylce Lanthanide Complexes in Solution Chirality 2002, 14, 562-567.

[24] Muller, G. Luminescent Chiral Lanthanide(III) Complexes as Potential Molecular Probes Dalton Trans. 2009, 9692-9707.

[25] Muller, G. Circularly Polarized Luminescence. In Luminescence of Lanthanide Ions; de Bettencourt-Dias, A., Ed.; John Wiley \& Sons, Inc.: Chichester, 2014 77-123. 
[26] Carr, R.; Evans, N. H.; Parker, D. LanthanideComplexes as Chiral Probes Exploiting Circularly Polarized Luminescence Chem. Soc. Rev. 2012, 41, 7673-7686.

[27] Zinna, F.; Di Bari, L. Lanthanide Circularly Polarized Luminescences: Bases and Applications Chirality 2015, 27, 1-13.

[28] Lunkley, J. L.; Shirotani, D.; Yamanari, K.; Kaizaki, S.; Muller, G. Extraordinary Circularly Polarized Luminescence Activity Exhibited by Cesium Tetrakis(3-Heptafluorobutylryl-(+)-Camphorato) $\mathrm{Eu}(\mathrm{III})$ Complexes in $\mathrm{EtOH}$ and $\mathrm{CHCl}_{3}$ Solutions J. Am. Chem. Soc. 2008, 130, 13814-13815.

[29] Lunkley, J. L.; Shirotani, D.; Yamanari, K.; Kaizaki, S.; Muller, G. Chiroptical Spectra of a Series of Tetrakis((+)-3-Heptafluorobutylrylcamphorato)lanthanide(III) with an Encapsulated Alkali Metal Ion: Circularly Polarized Luminescence and Absolute Chiral Structures for the Eu(III) and Sm(III) Complexes Inorg. Chem. 2011, 50, 12724-12732.

[30] Crawford, T. D. Ab Initio Calculation of Molecular Chiroptical Properties Theor. Chem. Acc. 2006, 115, 227-245.

[31] Autschbach, J. Computing chiroptical properties with first-principles theoretical methods: Background and illustrative examples Chirality 2009, 21, E116-E152.

[32] Longhi, G.; Castiglioni, E.; Koshoubu, J.; Mazzeo, G.; Abbate, S. Circularly Polarized Luminescence: A Review of Experimental and Theoretical Aspects Chirality 2016, 28, 697-707.

[33] Srebro-Hooper, M.; Autschbach, J. Calculating Natural Optical Activity of Molecules from First Principles Annu. Rev. Phys. Chem. 2017, 68, 399-420.

[34] Ianeselli, A.; Orioli, S.; Spagnolli, G.; Faccioli, P.; Cupellini, L.; Jurinovich, S.; Mennucci, B. Atomic Detail of Protein Folding Revealed by an Ab Initio Reappraisal of Circular Dichroism J. Am. Chem. Soc. 2018, 140, 3674-3682.

[35] Santoro, F.; Jacquemin, D. Going Beyond the Vertical Approximation with TimeDependent Density Functional Theory Wires Comput. Mol. Sci. 2016, 6, 460-486.

[36] Pritchard, B.; Autschbach, J. Calculation of Vibrationally Resolved Circularly Polarized Luminescence of d-Camphorquinone and (S,S)-trans-b-Hydrindanone ChemPhysChem 2010, 11, 2409-2415.

[37] Pecul, M.; Ruud, K. The optical activity of $\beta, \gamma$-enones in ground and excited states using circular dichroism and circularly polarized luminescence Phys. Chem. Chem. Phys. 2011, $13,643-650$.

[38] Schippers, P. H.; van de Ploeg, J. P. M.; Dekkers, H. P. J. Circular Polarization in the Fluorescence of $\beta, \gamma$-Enones: Distortion in the $\mathrm{n}^{1} \pi^{*}$ States J. Am. Chem. Soc. 1983, 105, 84-89. 
[39] McAlexander, H. R.; Crawford, T. D. Simulation of Circularly Polarized Luminescence Spectra using Coupled Cluster Theory J. Chem. Phys. 2015, 142, 154101.

[40] Autschbach, J. Spectroscopic Properties obtained from Time-Dependent Density Functional Theory (TD-DFT). In Computational Inorganic and Bioinorganic Chemistry, Vol. 9; Solomon, E. I.; Scott, R. A.; King, R. B., Eds.; John Wiley \& Sons: Chichester, UK, 2009 71-90.

[41] Autschbach, J. Calculating electronic optical activity of coordination compounds. In Comprehensive Inorganic Chemistry II, Vol. 9; Poeppelmeier, K.; Reedijk, J., Eds.; Elsevier: Oxford, UK, 2013 407-426.

[42] Autschbach, J.; Jorge, F. E.; Ziegler, T. Density functional calculations on electronic circular dichroism spectra of chiral cobalt(III) complexes Inorg. Chem. 2003, 42, 2867-2877.

[43] Jorge, F. E.; Autschbach, J.; Ziegler, T. On the Origin of the Optical Activity in the d-d Transition Region of Tris-bidentate Co(III) and Rh(III) Complexes Inorg. Chem. 2003, 42, 8902-8910.

[44] Fan, J.; Seth, M.; Autschbach, J.; Ziegler, T. Circular dichroism of trigonal dihedral Chromium(III) complexes: A theoretical study based on open-shell time-dependent density functional theory Inorg. Chem. 2008, 47, 11656-11668.

[45] Fan, J.; Ziegler, T. On the Origin of Circular Dichroism in Trigonal Dihedral $\mathrm{d}^{6}$ Complexes of Bidentate Ligands Containing Only $\sigma$-Orbitals. A Qualitative Model Based on a Density Functional Theory Study of $\Lambda-\left[\mathrm{Co}(\mathrm{en})_{3}\right]^{3+}$ Chirality 2008, 20, 938-950.

[46] Le Guennic, B.; Hieringer, W.; Görling, A.; Autschbach, J. Density functional calculations of electronic circular dichroism spectra of the transition metal complexes $\left[\mathrm{M}(\mathrm{phen})_{3}\right]^{2+}(\mathrm{M}$ = Fe, Ru, Os) J. Phys. Chem. A 2005, 109, 4836-4846.

[47] Rudolph, M.; Autschbach, J. Performance of conventional and range-separated hybrid density functionals in calculations of electronic circular dichroism spectra of transition metal complexes J. Phys. Chem. A 2011, 115, 14677-14686.

[48] Rudolph, M.; Autschbach, J. Calculation of Optical Rotatory Dispersion and Electronic Circular Dichroism for Tris-Bidentate group 8 and 9 metal complexes, with emphasis on exciton coupling J. Phys. Chem. A 2011, 115, 2635-2649.

[49] Mazzeo, G.; Fusè, M.; Longhi, G.; Rimoldi, I.; Cesarotti, E.; Crispini, A.; Abbate, S. Vibrational Circular Dichroism and Chiroptical Properties of Chiral Ir(III) Luminescent Complexes Dalton Trans. 2016, 45, 992-999.

[50] Karimova, N. V.; Aikens, C. M. Chiroptical Activity in BINAP- and DIOP-Stabilized Octa and Undecagold Clusters J. Phys. Chem. C 2018, 122, 11051-11065.

[51] Richardson, F. S.; Faulkner, T. R. Optical Activity of the f-f Transitions in Trigonal Diherdral $\left(\mathrm{D}_{3}\right)$ Lanthanide(III) Complexes. I. Theory J. Chem. Phys. 1982, 76, 1595-1606. 
[52] Saxe, J. D.; Faulkner, T. R.; Richardson, F. S. Optical Activity of the f-f Transitions in Trigonal Diherdral $\left(\mathrm{D}_{3}\right)$ Lanthanide(III) Complexes. II. Calculations J. Chem. Phys. 1982, 76, 1607-1623.

[53] Judd, B. R. Optical Absorption Intensities of Rare-Earth Ions Phys. Rev. 1962, 127, 750761.

[54] Ofelt, G. S. Intensities of Crystal Spectra if Rare-Earth Ions J. Chem. Phys. 1962, 37, 511520.

[55] Berardozzi, R.; Di Bari, L. Optical Activity in the Near-IR Region: The $\lambda=980 \mathrm{~nm}$ Multiplet of Chiral $\mathrm{Yb}^{3+}$ Complexes Chem. Phys. Chem. 2015, 16, 2868-2875.

[56] Wu, T.; Kessler, J.; Bour̃, P. Chiral Sensing of Amino Acids and Proteins Chelating with $\mathrm{Eu}^{I I I}$ Complexes by Raman Optical Activity Spectroscopy Phys. Chem. Chem. Phys. 2016, 18, 23803-23811.

[57] Jurinovich, S.; Pescitelli, G.; Di Bari, L.; Mennucci, B. A TDDFT/MMPol.PCM Model for the Simulation of Exciton-Coupled Circular Dichroism Spectra Phys. Chem. Chem. Phys. 2014, 16, 16407-16418.

[58] Berardozzi, R.; Pescitelli, G.; Di Pietro, S.; Resta, C.; Ballistreri, F. P.; Pappalado, A.; Tomaselli, G. A.; Di Bari, L. Synthesis, Structural Characterization, and Chiroptical Studies of Bidentate Salen-Type Lanthanide (III) Complexes Chirality 2015, 27, 857-863.

[59] Wu, T.; Hudecová, J.; You, X.-Z.; Urbanová, M.; Bour̃, P. Comparison of the Electronic and Vibrational Optical Activity of a Europium(III) Complex Chem. Eur. J. 2015, 21, 58075813.

[60] Kerridge, A. The Complete-Active-Space Self-Consistent-Field Approach and Its Application to Molecular Complexes if the f-Elements. In Computational Methods in Lanthanide and Actinide Chemistry; Dolg, M., Ed.; John Wiley \& Sons, Inc.: Chichester, 2015 121146.

[61] Roos, B. O.; Taylor, P. R.; Siegbahn, P. E. M. A Complete Active Space SCF method (CASSCF) using a density matrix formulated super-CI approach Chem. Phys. 1980, 48, $157-173$.

[62] Malmqvist, P.-A.; Roos, B. O.; Schimmelpfennig, B. The restricted active space (RAS) state interaction approach with spin-orbit coupling Chem. Phys. Lett. 2002, 357, 230-240.

[63] Frisch, M. J.; Trucks, G. W.; Schlegel, H. B.; Scuseria, G. E.; Robb, M. A.; Cheeseman, J. R.; Scalmani, G.; Barone, V.; Mennucci, B.; Petersson, G. A. et al. "Gaussian 09, Revision D.01”, Gaussian, Inc., Wallingford CT, 2009. URL: www.gaussian.com.

[64] Li, X.; Frisch, M. J. Energy-Represented Direct Inversion in the Iterative Subspace within a Hybrid Geometry Optimization Method J. Chem. Theory Comput. 2006, 2, 835-839. 
[65] Lee, C.; Yang, W.; Parr, R. G. Development of the Colle-Salvetti correlation-energy formula into a functional of the electron density Phys. Rev. B 1988, 37, 785-789.

[66] Becke, A. D. Density-functional thermochemistry. III. The role of exact exchange J. Chem. Phys. 1993, 98, 5648-5652.

[67] Yanai, T.; Handy, N. A New Hybrid Exchange-Correlation Functional Using the CoulombAttenuating Method Chem. Phys. Lett. 2004, 393, 51-57.

[68] Dunning Jr., T. H. Gaussian Basis Set for Use in Correlated Molecular Calculations. I. The Atoms Boron Through Neon and Hydrogen J. Chem. Phys. 1989, 90, 1007-1023.

[69] K., N.; Shiro, M.; Saito, Y.; Kuroya, H. Studies on Crystals of Metallic TrisEhtlyenediamine-Complexes. II. The Crystal Structure of Sodium D-Tris-EthylenediamineCobalt(III) chloride Hexahydrate, 2D-[Coen $\left.{ }_{3}\right] \mathrm{Cl}_{3} \mathrm{NaCl}_{6} 6 \mathrm{H}_{2} \mathrm{O}$ Bull. Chem. Soc. Jpn. 1956, $30,158-164$.

[70] Weigend, F.; Ahlrichs, R. Balanced Basis Sets of Split Valence, Triple Zeta Valence and Quadrupole Zeta Valence Quality for H to Rn: Design and Assessement of Accuracy Phys. Chem. Chem. Phys. 2005, 7, 3297-3305.

[71] Dolg, M.; Stoll, H.; Preuss, H. Energy-Adjusted Ab-Initio Pseudopotentials for the Rare Earth Elements J. Chem. Phys. 1989, 90, 1730.

[72] Cao, X.; Dolg, M. Segmented Contraction Scheme for Small-Core Lanthanide Pseudopotential Basis Sets J. Molec. Struct. Theochem 2002, 581, 139-147.

[73] Dunning Jr., T. H.; Hay, P. J. Gaussian Basis Sets for Molecular Calculations. In Methods of Electronic Structure Theory; Schaefer, H. F., Ed.; Springer US: New York, 1977 1-27.

[74] te Velde, G.; Bickelhaupt, F. M.; Baerends, E. J.; van Gisbergen, S. J. A.; Fonseca Guerra, C.; Snijders, J. G.; Ziegler, T. Chemistry with ADF J. Compt. Chem. 2001, 22, 931-967.

[75] Fonseca Guerra, C.; Snijders, J. G.; te Velde, G.; Baerends, E. J. Towards an order-N DFT method Theor. Chem. Acc. 1998, 99, 391.

[76] Baerends, E. J.; Ziegler, T.; Atkins, A. J.; Autschbach, J.; Bashford, D.; Baseggio, O.; Bérces, A.; Bickelhaupt, F. M.; Bo, C.; Boerritger, P. M. et al. "ADF2017, SCM, Theoretical Chemistry, Vrije Universiteit, Amsterdam, The Netherlands, https://www.scm.com”, 2017.

[77] Perdew, J. P.; Burke, K.; Ernzerhof, M. Generalized Gradient Approximation Made Simple Phys. Rev. Lett. 1996, 77, 3865-3868.

[78] Perdew, J. P.; Burke, K.; Ernzerhof, M. Generalized Gradient Approximation Made Simple [Phys. Rev. Lett. 77, 3865 (1996)] Phys. Rev. Lett. 1997, 78, 1396. 
[79] Ernzerhof, M.; Scuseria, G. E. Assessment of the Perdew-Burke-Ernzerhof exchangecorrelation functional J. Chem. Phys. 1999, 110, 5029-5036.

[80] Adamo, C.; Barone, V. Toward reliable density functional methods without adjustable parameters: The PBE0 model J. Chem. Phys. 1999, 110, 6158-6170.

[81] van Lenthe, E.; Baerends, E. J. Optimized Slater-type basis sets for the elements $1-118$ J. Comput. Chem. 2003, 24, 1142-1156.

[82] Autschbach, J.; Ziegler, T. Calculating molecular electric and magnetic properties from time dependent density functional perturbation theory J. Chem. Phys. 2002, 116, 891-896.

[83] Autschbach, J.; Ziegler, T.; Patchkovskii, S.; van Gisbergen, S. J. A.; Baerends, E. J. Chiroptical properties from time-dependent density functional theory. II. Optical rotations of small to medium sized organic molecules. J. Chem. Phys. 2002, 117, 581-592.

[84] van Gisbergen, S. J. A.; Snijders, J. G.; Baerends, E. J. Implementation of Time-Dependent Density Functional Response Equations Comput. Phys. Comm. 1999, 118, 119-138.

[85] van Lenthe, E.; Baerends, E. J.; Snijders, J. G. Relativistic regular two-component Hamiltonians J. Chem. Phys. 1993, 99, 4597-4610.

[86] van Lenthe, E.; Baerends, E. J.; Snijders, J. G. Relativistic Total Energy Using Regular Approximations J. Chem. Phys. 1999, 101, 9783.

[87] Pye, C. C.; Ziegler, T. An Implementation of the Conductor-Like Screening Model of Solvation wtihin the Amsterdam Density Functional Package Theor. Chem. Acc. 1999, 101, 396-408.

[88] Autschbach, J. Time-dependent density functional theory for calculating originindependent optical rotation and rotatory strength tensors ChemPhysChem 2011, 12, 32243235.

[89] Autschbach, J.; Ziegler, T.; van Gisbergen, S. J. A.; Baerends, E. J. Chiroptical properties from time-dependent density functional theory. I. Circular dichroism spectra of organic molecules. J. Chem. Phys. 2002, 116, 6930-6940.

[90] Martin, R. L. Natural Transition Orbitals J. Chem. Phys. 2003, 118, 4775-4777.

[91] Aquilante, F.; Autschbach, J.; Carlson, R. K.; Chibotaru, L. F.; Delcey, M. G.; Vico, L. D.; Fdez. Galván, I.; Ferré, N.; Frutos, L. M.; Gagliardi, L. et al. Molcas 8: New capabilities for multiconfigurational quantum chemical calculations across the periodic table J. Comput. Chem. 2016, 37, 506-541.

[92] Douglas, M.; Kroll, N. M. Quantum electrodynamical corrections to the fine structure of helium Ann. Phys. 1974, 82, 89-155.

[93] Hess, B. A. Applicability of the no-pair equation with free-particle projection operators to atomic and molecular structure calculations Phys. Rev. A 1985, 32, 756-763. 
[94] Hess, B. A. Relativistic electronic-structure calculations employing a two-component nopair formalism with external-field projection operators Phys. Rev. A 1986, 33, 3742-3748.

[95] Wolf, A.; Reiher, M.; Hess, B. A. The generalized Douglas-Kroll transformation J. Chem. Phys. 2002, 117, 9215-9226.

[96] Widmark, P.-O.; Malmqvist, P.-Å.; Roos, B. O. Density-matrix averaged atomic natural orbital (ANO) basis-sets for correlated molecular wave-functions. I. First row atoms Theor. Chim. Acta 1990, 77, 291-306.

[97] Roos, B. O.; Lindh, R.; Malmqvist, P.-Å; Veryazov, V.; Widmark, P.-O. Main group atoms and dimers studied with a new relativistic ANO basis set J. Phys. Chem. A 2004, 108, 2851-2858.

[98] Roos, B. O.; Lindh, R.; Malmqvist, P.- $\AA$.; Veryazov, V.; Widmark, P.-O. New relativistic ANO basis sets for transition metal atoms J. Phys. Chem. A 2005, 109, 6575.

[99] Andersson, K.; Malmqvist, P.-Å.; Roos, B. O.; Sadlev, A. J.; Wolinski, K. Second-order perturbation theory with a CASSCF reference function J. Phys. Chem. 1990, 94, 54835488.

[100] Cossi, M.; Rega, N.; Scalmani, G.; Barone, V. Polarizable Dielectric Model of Solvation with Inclusion of Charge Penetration Effects J. Chem. Phys. 2001, 114, 5691.

[101] Barone, V.; Cossi, M. Quatum Calculation of Molecular Energies and Energy Gradients in Solution by a Conductor Solvent Model J. Phys. Chem. A 1998, 102, 1995-2001.

[102] Gendron, F.; Fleischauer, V. E.; Duignan, T. J.; Scott, B. L.; Löble, M. W.; Cary, S. K.; Kozimor, S.; Bolvin, H.; Neidig, M. L.; Autschbach, J. Magnetic circular dichroism of $\mathrm{UCl}_{6}^{-}$in the ligand-to-metal charge-transfer spectral region Phys. Chem. Chem. Phys. 2017, 19, 17300-17313.

[103] Bolvin, H. $\mathrm{d} \rightarrow \mathrm{d}$ Spectrum and High-Spin/Low-Spin Competition in $\mathrm{d}^{6}$ Octahedral Coordination Compounds: Ab Initio Study of Potential Energy Curves J. Phys. Chem. A 1998, 102, 7525-7534.

[104] Kepenekian, M.; Robert, V.; Le Guennic, B. What Zroth-Order Hamiltonian for CASPT2 Adiabatic Energestics of Fe(II)N ${ }_{6}$ Architectures ? J. Chem. Phys. 2009, 131, 114702.

[105] Vela, S.; Fumanal, M.; Ribas-Ariño, J.; Robert, V. On the Zeroth-Order Hamiltonian for CASPT2 Calculations of Spin Crossover Compounds J. Comput. Chem. 2016, 37, 947-953.

[106] Lestrange, P. J.; Egidi, F.; Li, X. The Consequences of Improperly Describing Oscillator Strengths beyond the Electric Dipole Approximation J. Chem. Phys. 2015, 143, 234103.

[107] Sørensen, L. K.; Guo, M.; Lindh, R.; Lundberg, M. Applications to metal K pre-edges of transition metal dimers illustrate the approximate origin independence for the intensities in the length representation Mol. Phys. 2017, 115, 174-189. 
[108] Pedersen, T. B.; Hansen, A. E. Ab-initio Calculation and Display of the Rotatory Strength Tensor in the Random Phase Approximation. Method and Model Studies Chem. Phys. Lett. 1995, 246, 1-8.

[109] DeBeer George, S.; Petrenko, T.; Neese, F. Time-Dependent Density Functional Calculations of Ligand K-Edge X-ray Absorption Spectra Inorg. Chim. Acta 2008, 361, 965-972.

[110] Longhi, G.; Castiglioni, E.; Abbate, S.; Lebon, F.; Lightner, D. A. Experimental and Calculated CPL Spectra and Related Spectroscopic Data of Camphor and Other Simple Chiral Bicyclic Ketones Chirality 2013, 25, 589-599.

[111] Autschbach, J.; Srebro, M. Delocalization error and 'functional tuning' in Kohn-Sham calculations of molecular properties Acc. Chem. Res. 2014, 47, 2592-2602.

[112] Emeis, C. A.; Oosterhoff, L. J. Emission of Circularlay Polarized Radiation by Optically Active Compounds Chem. Phys. Lett. 1967, 1, 129-132.

[113] Emeis, C. A.; Oosterhoff, L. J. The $n-\pi^{*}$ Absorption and Emission of Optically Active trans- $\beta$-Hydrindanone and trans- $\beta$-Thiohydrindanone J. Chem. Phys. 1971, 54, 48094819.

[114] Viswanatha, C. b.; Helmich-Paris, B.; Hättig, C. Circularly polarized fluorescence and phosphorescence calculations on organic molecules using the approximate coupled-cluster model CC2 Phys. Chem. Chem. Phys. 2018, 20, 21051-21061.

[115] Moore, B. I.; Srebro, M.; Autschbach, J. Analysis of Optical Activity in Terms of Bonds and Lone-Pairs: The exceptionally LArge Optixal Roatation of Norbornenone J. Chem. Theory Comput. 2012, 8, 4336-4346.

[116] Caricato, M. Orbital Analysis of Molecular Optical Activity Based on Configuration Rotatory Strength J. Chem. Theory Comput. 2015, 11, 1349-1353.

[117] Caricato, M.; Vaccaro, P. H.; Crawford, T. D.; Wiberg, K. B.; Lahiri, P. Insights on the Origin of the Unusually Large Specific Rotation of (1S,4S)-Norbornenone J. Phys. Chem. A 2014, 118, 4863-4871.

[118] Kobayshi, M. Optical Rotatory Power and Circular Dichroism. III. Rotatory Dispersion of D-[Coen $\left.{ }_{3}\right] \mathrm{Br}_{3}$ J. Chem. Soc. Jpn. 1943, 64, 648-653.

[119] Mathieu, J.-P. Circular Dichroism, Valence Bonds, and Secondary Asymmetric Sybthesis Ann. Phys. 1944, 19, 335-354.

[120] Mason, S. F.; Peart, B. J. Crystal Circular Dichroism and Spin-Forbidden Optical Activity of Tris(Diamine)Cobalt(III) Complexes J. C. S. Dalton Trans. 1977, 0, 937-941.

[121] Strickland, R. W.; Richardson, F. S. Optical Activity of Transition Metal Compounds. III. Molecular Orbital Calculations on Six-Coordinate Complexes of Trigonal Symmetry Inorg. Chem. 1973, 12, 1025-1036. 
[122] Evans, R. S.; Schreiner, A. F.; Hauser, P. J. Natural Optical Activity of Co(en $)_{3}{ }^{3+}$ and $\mathrm{Cr}(\mathrm{en})_{3}{ }^{3+}:$ Interpretations with Complete Operator Matrices Inorg. Chem. 1974, 13, $2185-$ 2192.

[123] Mason, S. F.; Seal, R. H. Complementation of the Crystal-Field for Dihedral Optical Activity J. C. S. Chem. Comm. 1975, 0, 331-333.

[124] Mason, S. F.; Seal, R. H. The Optical Activity of Cobalt(III) Chelate Diamine Complexes Mol. Phys. 1976, 31, 755-775.

[125] Moffit, W. Optical Rotatory Dispersion of Transition-Metal Complexes J. Chem. Phys. 1956, 25, 1189-1198.

[126] Lehr, A. D. Interaction of Electromagnetic Radiationwith Matter. I. Theory of Optical Rotatory Power: Topic A. Trigonal Dihedral Compounds J. Chem. Phys. 1964, 68, 665-722.

[127] Karipides, A. G.; Piper, T. S. Optical Activity of Coordination Compounds. II. A Molecular Orbital Model and an Analysis of Experimental Data fir Complexes of Trigonal Symmetry J. Chem. Phys. 1964, 40, 674-682.

[128] Richardson, F. S. The Optical Activity of Trigonally Distorded Cubic Systems J. Phys. Chem. 1971, 75, 692-712.

[129] Baron, L. D. Electric Quadrupole Contributions to the Optical Activity of Crystalline Transition Metal Complexes Mol. Phys. 1971, 21, 241-246.

[130] Ernst, M. C.; Royer, D. J. Optical Activity in Tris Bidentate Cobalt(III) Complexes. An ab initio Study Inorg. Chem. 1993, 32, 1226-1232.

[131] Jorge, F. E.; Autschbach, J.; Ziegler, T. On the Origin of Optical Activity in tris-diamine complexes of Co(III) and Rh(III). A Simple Model based on Time-dependent Density Function Theory J. Am. Chem. Soc. 2005, 127, 975-985.

[132] Kuroda, R.; Saito, Y. Solid-State Circular Dichroism Spectra of Tris(diamine)cobalt(III) Complexes: Decomposition in E and $\mathrm{A}_{2}$ Components Bull. Chem. Soc. Jpn. 49, 433-436.

[133] Richardson, F. S. The Optical Activity of Disymmetric Six-Coordinate Cobalt(III) Complexes Inorg. Chem. 1972, 11, 2366-2378.

[134] Kamiński, M.; Cukras, J.; Pecul, M.; Rizzo, A.; Coriani, S. A Computational Protocol for the Study of Circularly Polarized Phosphorescence and Circular Dichroism in SpinForbidden Absorption Phys. Chem. Chem. Phys. 2015, 17, 19079-19086.

[135] Eliseeva, S. V.; Bünzli, J.-C. G. Lanthanide Luminescence for Functional Materials and Bio-Sciences Chem. Soc. Rev. 2010, 39, 189-227.

[136] D’Aléo, A.; Picot, A.; Beeby, A.; Williams, J. A. G.; Le Guennic, B.; Andraud, C.; Maury, O. Efficient Sensitization of Europium, Ytterbium, and Neodymium Functionalized Tris-Dipicolinate Lanthanide Complexes Through Tunable Charge-Transfer Excited States Inorg. Chem. 2008, 47, 10258-19268. 
[137] Janicki, R.; Kedziorski, A.; Mondry, A. The First Example of ab-initio Calculations of f-f Transitions for the Case of $[\mathrm{Eu}(\mathrm{DOTP})]^{5-}$ Complex - Experiment versus Theory Phys. Chem. Chem. Phys. 2016, 18, 27808-27817.

[138] Freidzon, A. Y.; Kurbatov, I.; Vovna, V. I. Ab inito Calculation of Energy Levels of Trivalent Lanthanide Ions Phys. Chem. Chem. Phys. 2018, 20, 14564.

[139] Hatanaka, M.; Yabushita, S. Mechanisms of f-f Hypersensitive Transition Intensities of Lanthanide Trihalide Molecules: A Spin-Orbit Conifguration Interaction Study Theor. Chem. Acc. 2014, 133, 1517.

[140] Hilmes, G. L.; Riehl, J. P. Circularly Polarized Luminescence from Racemic Lanthanide(III) Complexes with Achiral Ligands in Aqueous Solution using Circularly Polarized Excitation Inorg. Chem. 1986, 25, 2617-2622.

[141] Richardson, F. S. Terbium(III) and Europium(III) Ions as Luminescent Probes and Stains for Biomolecular Systems Chem. Rev. 1982, 82, 541-552.

[142] Werts, M. H. V.; Jukes, R. T. F.; Verhoeven, J. W. The Emission Spectrum and the Radiative Lifetome of $\mathrm{Eu}^{3+}$ in Luminescent Lanthanide Complexes Phys. Chem. Chem. Phys. 2002, $4,1542-1548$.

[143] Binnemans, K. Interpretation of Europieum(III) Spectra Coord. Chem. Rev. 2015, 295, 145.

[144] Huskowska, E.; Gawryszewska, P.; Legendziewicz, J.; Maupin, C. L.; Riehl, J. The Measurement of Circularly Polarized Luminescence from Racemic Lanthanide Complexes Prepared in Sol-Gels J. Alloys Compd. 2000, 303, 325-330.

[145] Sokolnicki, J.; Legendziewicz, J.; Riehl, J. P. The Effect of Excited State Energy Transfer on the Circularly Polarized Luminescence from Sol-Gels Containing Racemic Complexes of Eu(III): Theory and Experiment J. Phys. Chem. B 2002, 106, 1508-1514.

[146] Sokolnicki, J.; Legendziewicz, J.; Muller, G.; Riehl, J. P. The Luminescence, Molecular and Electronic Structure, and Excited State Energetics of Tris-Complexes of 4phenylethynil-2,6-pyridinedicarboxylic Acid with Eu(III) and Tb(III) Prepared in Sol-Gel Opt. Mat. 2005, 27, 1529-1536.

[147] Coruh, N.; Hilmes, G. L.; Riehl, J. P. Use of the Pfeiffer Effect to Probe the Optical Activity of Europium(III) Complexes with 2,6-Pyridinedicarboxylate Inorg. Chem. 1988, 27, 3647-3651.

[148] Knecht, S.; Keller, S.; Autschbach, J.; Reiher, M. A nonorthogonal state-interaction approach for matrix product state wave functions J. Chem. Theory Comput. 2016, 12, 58815894.

[149] Keller, S. F.; Reiher, M. Determining Factors for the Accuracy of DMRG in Chemistry Chimia 2014, 68, 200-203. 


\section{Table of Contents Graphics}

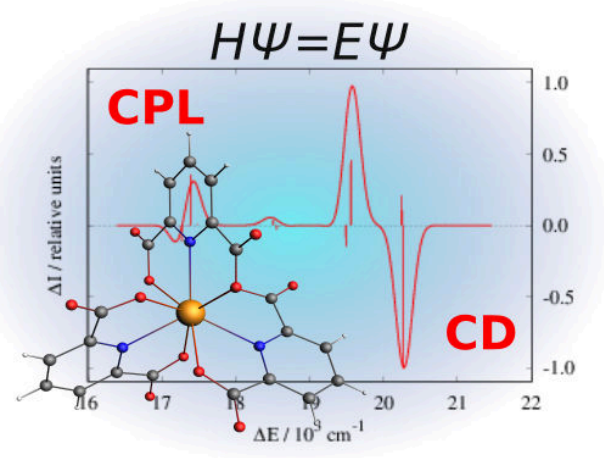

\section{Table of Contents Synopsis}

The absorption CD and emission CPL properties of a series of four bicyclic ketones, the $\Lambda$ $\left[\mathrm{Co}(\mathrm{en})_{3}\right]^{3+}$ complex, and of the $\Delta$ - and $\Lambda$-[Eu(DPA $\left.)_{3}\right]^{3-}$ enantiomers are calculated with the help of multi-reference wavefunction calculations including spin-orbit coupling that allow to treat both spin-allowed and spin-forbidden transitions.

\section{Dedication}

This article is dedicated to Dr. Jean-Paul Malrieu on the occasion of his 80th birthday. 